\title{
Simulations of heavy-ion halo collimation at the CERN Large Hadron Collider: Benchmark with measurements and cleaning performance evaluation
}

\author{
N. Fuster-Martínez $\oplus^{*}$ R. Bruce, F. Cerutti, R. De Maria, P. Hermes, A. Lechner, \\ A. Mereghetti, J. Molson $\odot$, S. Redaelli, and E. Skordis \\ CERN, Geneva, Switzerland \\ A. Abramov $\odot$ and L. Nevay \\ John Adams Institute at Royal Holloway, University of London, Egham, TW20 OEX, United Kingdom
}

(Received 27 June 2020; accepted 14 October 2020; published 6 November 2020)

\begin{abstract}
Protons and heavy-ion beams at unprecedented energies are brought into collisions in the CERN Large Hadron Collider (LHC) for high-energy experiments. The LHC multistage collimation system is designed to provide protection against regular and abnormal losses in order to reduce the risk of quenches of the superconducting magnets as well as keeping background in the experiments under control. Compared to protons, beam collimation in the heavy-ion runs is more challenging despite the lower stored beam energies, because the efficiency of cleaning with heavy ions has been observed to be 2 orders of magnitude worse. This is due to the differences in the interaction mechanisms between the beams and the collimators. Ion beams experience fragmentation and electromagnetic dissociation at the collimators that result in a substantial flux of off-rigidity particles that escape the collimation system. These out-scattered nuclei might be lost around the ring, eventually imposing a limit on the maximum achievable stored beam energy. The more stringent limit comes from potential quenches of superconducting magnets. Accurate simulation tools are crucial in order to understand and control these losses. A new simulation framework has been developed for heavy-ion collimation based on the coupling of the SIXTRACK tracking code, which has been extended to track arbitrary heavy-ion species, and the FLUKA Monte Carlo code that models the electromagnetic and nuclear interactions of the heavy ions with the nuclei of the collimator material. In this paper, the functionality of the new simulation tool is described. Furthermore, SIXTRACK-FLUKA coupling simulations are presented and compared with measurements done with ${ }^{208} \mathrm{~Pb}^{82+}$ ions in the LHC. The agreement between simulations and measurements is discussed and the results are used to understand and optimize losses. The simulation tool is also applied to predict the performance of the collimation system for the highluminosity LHC. Based on the simulation results and the experience gained in past heavy-ion runs, some conclusions are presented.
\end{abstract}

DOI: 10.1103/PhysRevAccelBeams.23.111002

\section{INTRODUCTION}

At the CERN Large Hadron Collider (LHC) [1], proton and heavy-ion beams are brought into collision for highenergy physics experiments. Unavoidable losses occur in colliders due to the interaction of the main beam with residual gas in the beam pipe, the collision of the beams at the interaction points, instabilities, resonances, or due to the diffusion mechanisms driven by electron cloud and beam-beam interactions, just to name a few. The LHC

*nuria.fuster.martinez@cern.ch

Published by the American Physical Society under the terms of the Creative Commons Attribution 4.0 International license. Further distribution of this work must maintain attribution to the author(s) and the published article's title, journal citation, and DOI. multistage collimation system [2-6] is designed to protect the LHC hardware against regular and abnormal beam losses. In particular, the collimation system has to protect the superconducting magnets that risk quenching, changing their state from superconducting to normal conducting, reducing as a consequence the available time for physics data acquisition. In addition, the collimation system also has to keep the background in the experiments under control $[7,8]$. For heavy-ion beams a degraded collimation cleaning efficiency is expected. This is due to the nuclear fragmentation and electromagnetic dissociation processes occurring at the collimators that generate a large spectrum of secondary nuclei with a different charge-to-mass ratio with respect to the main beam. Some of these fragments can escape the downstream collimation stages and be lost at other locations around the ring [9-12]. In order to evaluate the performance of the collimation system the collimation cleaning efficiency is calculated as the sum of the energy 


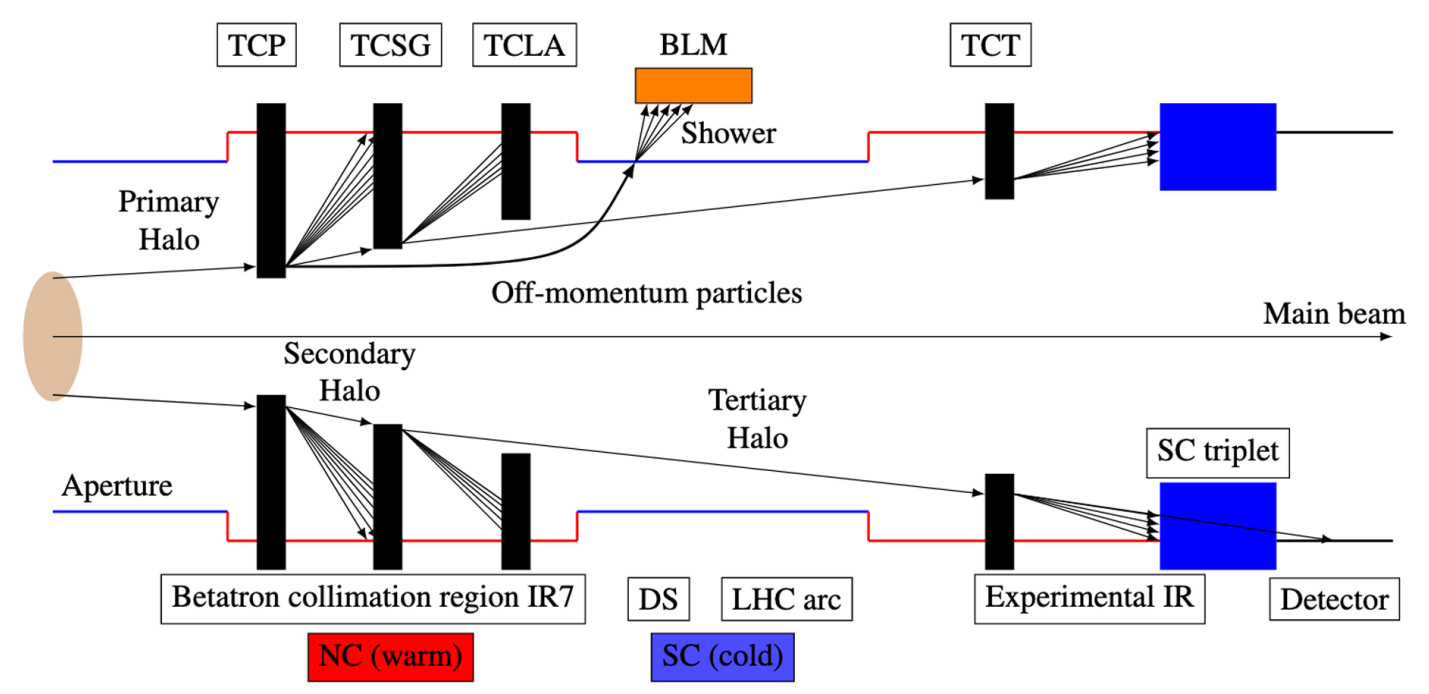

FIG. 1. LHC multistage collimation system scheme where the different collimator families are indicated. Normal conducting (NC) and superconducting (SC) apertures are depicted in red and blue, respectively [12].

lost at a given location, $s$, per unit length, normalized by the maximum energy lost in the ring within a distance $\Delta s$, $E_{\max }$, as

$$
\eta(s)=\frac{\sum_{i} E_{i}(s)}{\Delta s E_{\max }}
$$

where $E_{i}$ is the energy of the ion $i$ lost within a distance $\Delta s$ around the position $s$. In the LHC heavy-ion runs, a reduction by a factor 100 of the collimation cleaning efficiency has been observed in comparison to protons, which is not fully compensated by the lower stored beam energies reached during these runs [13-16]. This makes the collimation of heavy ions, which typically are operated during one month per year, more challenging.

In $2018,{ }^{208} \mathrm{~Pb}^{82+}$ ion beams were accelerated to an energy of $6.37 \mathrm{ZTeV}^{1}$ in the LHC [17]. The stored beam energy reached by the ions was $13.3 \mathrm{MJ}$, which is well above the design value of $3.8 \mathrm{MJ}$ [1] and what was previously achieved at the LHC [15,18]. In the 2018 ${ }^{208} \mathrm{~Pb}^{82+}$ ion run, no magnet quenches were recorded due to collimation losses from circulating beams, but seven out of 48 fills were dumped due to high losses in collimators caused by orbit oscillations [19]. These beam dumps could be avoided by a better collimation cleaning efficiency. This underlines the need for a solution for future runs where even higher intensities are envisaged by the high-luminosity LHC project (HL-LHC) [20].

The development of accurate simulation tools is crucial to understand and control the secondary fragments generated at the collimators, quantify possible limitations, and elaborate mitigation strategies for future runs. Significant progress has been made in the past years to improve the accuracy of the heavy-ion collimation simulation tools. In this paper, a new heavy-ion simulation tool developed

\footnotetext{
${ }^{1} Z$ is the charge number of the nuclei in the main beam.
}

based on the coupling of the SixTRACK tracking code [21,22] and the FLUKA [23,24] Monte-Carlo program, similar to the development of simulation tools for protons $[25,26]$ is presented. In addition, the performance of the LHC collimation system, simulated with the SIXTRACKFLUKA coupling tool, is presented and compared to measurements for different scenarios for a better understanding and optimization of losses in the LHC. Furthermore, using this new tool, the performance in future configurations is predicted. The paper is organized as follows. First, the LHC collimation system is described in Sec. II as well as the measurements performed at the start of every run to qualify the performance of the collimation system. These measurements are later used for the comparison with simulations. In Sec. III the heavy-ion collimation simulation state-of-the-art tools are presented and the functionality of the SIXTRACK-FLUKA coupling framework is described. In Sec. IV, the methodology followed to perform the analysis of the simulations and the simulation setup are described. In Sec. V, SiXTRACK-FLUKA coupling simulations are presented and compared to measurements from the $2018{ }^{208} \mathrm{~Pb}^{82+}$ ion run, and operational applications are discussed. In addition, detailed simulations performed with FLUKA and more complete geometries are compared to measurements performed in the 2015 ${ }^{208} \mathrm{~Pb}^{82+}$ ion run and the agreement is discussed. In the last section, the simulation tool is used to predict the cleaning performance of the collimation system for the future upgraded HL-LHC configuration.

\section{HEAVY-ION BEAM COLLIMATION AT THE LHC}

The LHC multistage collimation system is organized in a well-defined hierarchy, based on their opening, with different collimator families as illustrated in Fig. 1, where 


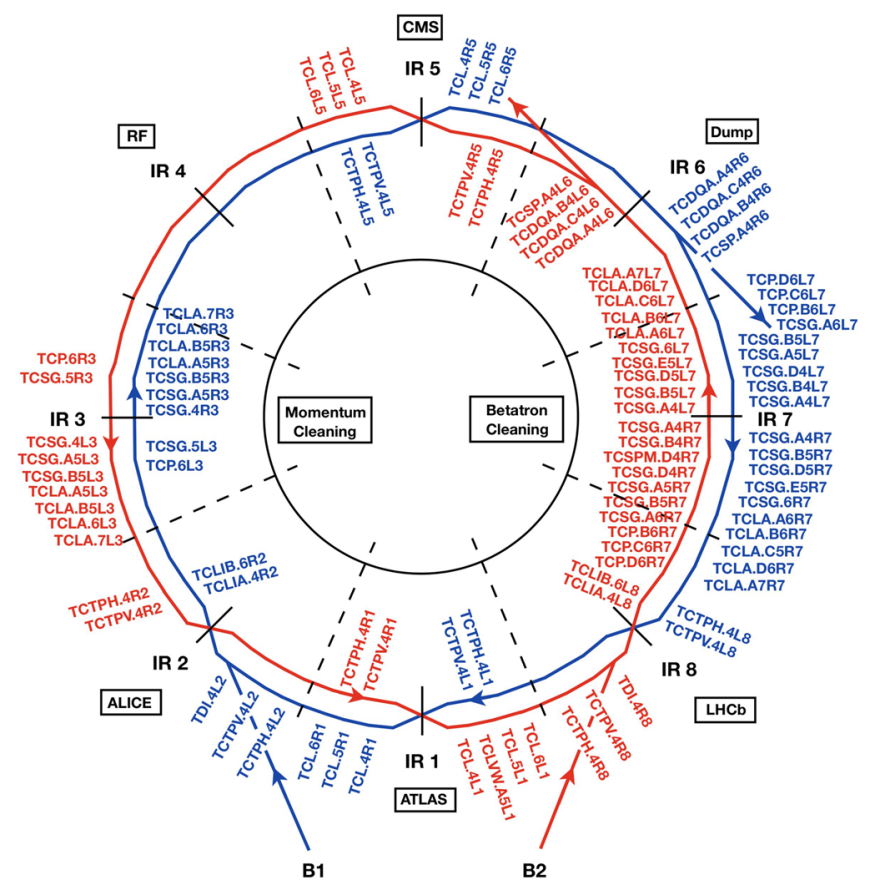

FIG. 2. 2018 LHC collimation system layout [31] for B1 (in blue) and B2 (in red). The names and locations of the different IRs are also indicated.

each individual collimator consists of two movable jaws with the beam passing in the center. The preservation of the hierarchy between families is a prerequisite to ensure a good performance of the system.

The first family is made of primary collimators (TCPs), which are the closest ones to the beams and have their jaws made of carbon-fiber composite (CFC). The second family is composed of secondary collimators (TCSGs), also made of CFC, followed by the active absorbers (TCLAs), made of Inermet-180 (heavy tungsten alloy), which are placed to absorb particles out-scattered by the TCPs (secondary and tertiary beam halo). These collimators are installed in two insertion regions (IRs): IR7 for betatron cleaning and IR3 for off-momentum cleaning. Then, tertiary collimators (TCTs) made of Inermet-180, are installed upstream of the experimental IRs. These collimators aim to absorb the tertiary betatron beam halo and to provide passive protection of the aperture of the triplet quadrupoles of the final focusing system, as well as to control the experimental backgrounds. Downstream of the experiments other tertiary collimators are installed to absorb the debris from the collisions (TCLs). Furthermore, two other CFC collimators per beam (TCSP, TCDQ) are installed in the extraction region (IR6) for beam dump protection. These collimators must ensure the protection of the machine in case of beam dump failures (BDFs) [27-30]. The full 2018 LHC collimation layout is depicted in Fig. 2 for the two counterrotating LHC beams called beam 1 (B1) in blue and beam 2 (B2) in red.
The LHC operational cycle consists of different processes. The beam is injected into the ring with an energy of $450 \mathrm{ZGeV}$. During the "ramp and squeeze" the beam energy is increased up to its maximum of the run while the $\beta$ functions at the collision points $\left(\beta^{*}\right)$ are decreased, which is called "squeeze." Once at top energy (this static point in the cycle is referred to as "flattop" in the following) the squeeze continues in a separate process to reach the minimum $\beta^{*}$, which is possibly different for the four experimental points. Finally, in the last process that takes place after the end of the squeeze, the beams are brought in collision in the experiments in IR1/2/5/8 (see Fig. 2), the end point of this process is called "physics."

Before high-intensity beams are allowed in the machine, the performance of the collimation system is validated. This is done at each static point of the LHC cycle (injection, flattop, end of squeeze, and physics) by deliberately inducing losses using a safe, low-intensity beam and observing the resulting loss pattern. For the betatron cleaning, losses are induced by blowing up the beam in the transverse planes with the transverse damper that can inject bandlimited white noise in the beam [32]. For the offmomentum cleaning validation, losses are induced by shifting the frequency of the radio frequency (rf) system. The losses occurring around the ring are recorded by beam loss monitors (BLMs) [33,34]. Then, the BLM signals are plotted as a function of the location in the ring, $s$, and the losses are classified as cold (blue), warm (red) or collimator (black). The cold losses refer to losses in the aperture of SC magnets while the warm losses refer to losses in NC magnets and other equipment at room temperature. For the purpose of evaluating the collimation cleaning, the BLM signals are normalized by the highest BLM signal measured in the ring, which is typically measured in IR7 where primary beam losses are intercepted. The resulting loss distribution is called a loss map. These loss maps have been used to evaluate the agreement between measurements and simulations performed with the new SIXTRACK-FLUKA coupling simulation tool.

As an example, in Fig. 3 the 2018 full ring [3(a)] and IR7 [3(b)] horizontal betatron loss maps for B1 are shown for protons (top) and ${ }^{208} \mathrm{~Pb}^{82+}$ ions (bottom). These loss maps were performed with colliding beam optics. The highest cold spikes are found in three clusters downstream of the collimation system in the dispersion suppressors (DS) indicated in Fig. 3 as DS1 $(s=1150-1210 \mathrm{~m})$, DS2 $(s=1230-1300 \mathrm{~m})$ and $\operatorname{DS} 3(s=1390-1410 \mathrm{~m})$. As can be seen in Fig. 3 the collimation cleaning efficiency in the three cold clusters in IR7 worsens by 2 orders of magnitude for ${ }^{208} \mathrm{~Pb}^{82+}$ ions as compared to protons. This worsening in the DS clusters is dominated by the energy lost by the heaviest ion fragments produced in the primary collimators such as ${ }^{207} \mathrm{~Pb}$ and ${ }^{206} \mathrm{~Pb}$, mainly because they have among the highest production cross sections and a total energy deviation with respect to the reference ion 

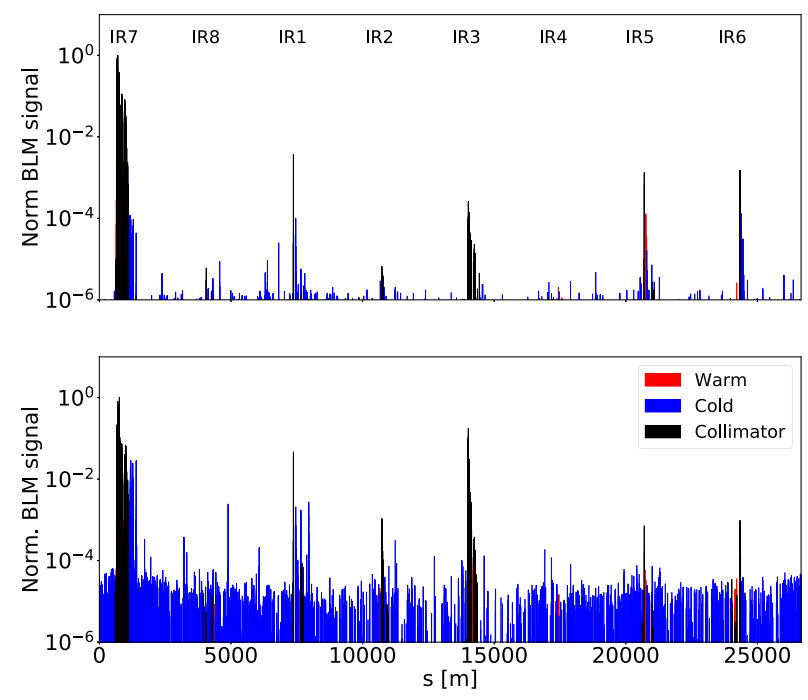

(a)

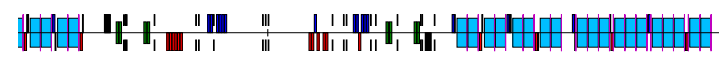
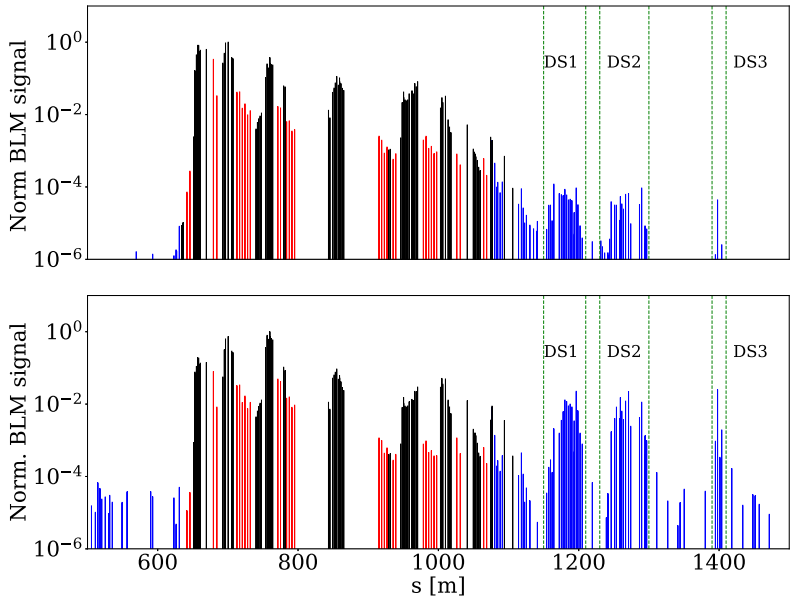

(b)

FIG. 3. Measured horizontal B1 full ring (a) and IR7 zoom (b) loss maps performed with colliding $6.5 \mathrm{TeV}$ proton (top) and $6.37 \mathrm{Z} \mathrm{TeV}^{208} \mathrm{~Pb}^{82+}$ ion (bottom) beams. In the IR7 zoom, the three clusters in the DS in which the highest cold loss spikes around the ring are observed, are indicated as DS1 $(s=1150-1210 \mathrm{~m})$, DS2 $(s=1230-1300 \mathrm{~m})$ and DS3 $(s=1390-1410 \mathrm{~m})$. Note that the layout has been started in IR7 with the vertical TCP located at $s=650 \mathrm{~m}$. On the top of (b) the different layout elements are indicated: dipoles (light blue), focusing quadrupoles (blue), defocusing quadrupoles (red), sextupoles (green) and collimators (black).

species that is small enough to reach the cold magnets in the DS where the acceptance is expected to be about $1 \%$.

In the LHC, the validation of the collimation system performance is completed with asynchronous beam dump failure (ABDF) tests, which is a type of BDF scenario, in order to validate the protection of the machine by the collimation system during such failures. This critical failure occurs when there is a dump of the beam out of synchronization with the abort gap [gap without beam that allows the extraction kicker magnets (MKDs) to rise up to full field]. Miskicked bunches could cause fast high losses with consequent risk of damage of sensitive components. The most exposed elements are the collimators made of tungsten (TCTs and TCLAs) and the triplets that need to stay sufficiently in the shadow of the TCDQ and the TCSP collimators. These measurements are also an essential part of the beam commissioning after long periods without beam or following relevant changes in the hardware or in the machine configuration. For these tests, a single bunch is injected close to the abort gap and the orbit is bumped away from the TCDQ to simulate the maximum allowed orbit excursion in the extraction region. Then, the rf is switched off, thus allowing the beam to debunch and drift into the abort gap. This is monitored and when the beam fills the abort gap the beam is dumped by the operators. The resulting loss maps generated are analyzed to evaluate the performance of the collimation system in such failure scenarios. This type of loss map has also been used to benchmark the simulation framework presented in this paper.
Data from two different heavy-ion runs are used in this work. The first set of data is from the most recent $\mathrm{Pb}-\mathrm{Pb}$ run in 2018 at the energy of $6.37 \mathrm{Z} \mathrm{TeV} \mathrm{[17].} \mathrm{The} \mathrm{second} \mathrm{set} \mathrm{of}$ data used in the study is from a SC magnet quench test performed on the 13th of December 2015 with ${ }^{208} \mathrm{~Pb}^{82+}$ ions $[14,35]$. This test was performed in order to measure the quench limit of the SC magnets in IR7, which are expected to quench with an instantaneous power load of $15 \mathrm{~kW}$. In such measurements, very high losses were produced at the primary collimator using the transverse damper, with the aim of quenching the IR7 DS magnets with the collimator debris in a controlled manner. B2 and the horizontal plane were used for the experiment in which, for the first time in the LHC, a magnet quench was achieved with beam losses from collimators in a controlled way. The better understanding of the quench limit and agreement with expectations allows pushing the maximum stored beam energy in the machine and defines upgrade requirements for the LHC collimation system. These measurements offered a unique opportunity to benchmark simulations and measurements with very high resolution as the high losses cause a high signal-to-noise ratio. The main beam parameters and collimator settings for both runs are summarized in Tables I and II.

To calculate the collimator settings in units of beam size, $\sigma$, the normalized LHC design emittance for protons, $\epsilon_{N}^{P}=3.5 \times 10^{-6} \mathrm{mrad}$ is used. In order to define comparable collimator settings for the ion beams the same geometrical emittance has to be used. Therefore an equivalent normalized ion emittance $\epsilon_{N}^{I}$ is calculated taking into 
TABLE I. $\quad$ B1 2015 and $2018{ }^{208} \mathrm{~Pb}^{82+}$ main optics and beam run parameters at flattop and physics static points of the LHC cycle.

\begin{tabular}{lcccc}
\hline \hline & & ${ }^{208} \mathrm{~Pb}^{82+}$ & ${ }^{208} \mathrm{~Pb}^{82+}$ & ${ }^{208} \mathrm{~Pb}^{82+}$ \\
\cline { 3 - 5 } & Units & 2018 flattop & 2018 physics & 2015 quench test \\
\hline$\beta^{*}$ IR1/2/5/8 & {$[\mathrm{m}]$} & $1 / 1 / 1 / 1.5$ & $0.5 / 0.5 / 0.5 / 1.5$ & $1 / 1 / 1 / 3$ \\
Half-crossing angle IR1/2/5/8 & {$[\mu \mathrm{rad}]$} & $160 / 200 / 160 /-170$ & $160 / 137 / 160 /-170$ & $-145 / 137 / 145 /-250$ \\
Beam separation IR1/2/5/8 & {$[\mathrm{mm}]$} & $1.1 / 6 / 1.1 / 2$ & $0 / 0 / 0 / 0$ & $1.1 / 4 / 1.1 / 2$ \\
IP shift IR1/2/5/8 & {$[\mathrm{mm}]$} & $0 / 0 / 0 / 0$ & $0 /-2 /-1.8 / 0$ & $0 / 0 / 0 / 0$ \\
$\mathrm{E}$ & {$[\mathrm{Z} \mathrm{TeV}]$} & 6.37 & 6.37 & 6.37 \\
$\epsilon_{N}$ & {$\left[\times 10^{-6} \mathrm{~m} \mathrm{rad}\right]$} & 1.39 & 1.39 & 1.39 \\
\hline \hline
\end{tabular}

TABLE II. 2015 and $2018{ }^{208} \mathrm{~Pb}^{82+}$ ion runs collimator settings for $\epsilon_{N}^{P}=3.5 \mu \mathrm{m}$. L and R indicates the left and right jaw, respectively. $\mathrm{H}$ and $\mathrm{V}$ correspond to horizontal and vertical planes, respectively.

\begin{tabular}{|c|c|c|c|c|c|}
\hline \multirow[b]{3}{*}{ Collimator } & \multirow[b]{3}{*}{ Beam } & \multirow[b]{3}{*}{ IR } & \multirow{2}{*}{$\frac{{ }^{208} \mathrm{~Pb}^{82+} 2018 \text { flattop }}{\left(\beta^{*}=100 \mathrm{~cm}\right)}$} & \multirow{2}{*}{$\frac{{ }^{208} \mathrm{~Pb}^{82+} 2018 \text { physics }}{\left(\beta^{*}=50 \mathrm{~cm}\right)}$} & \multirow{2}{*}{$\frac{{ }^{208} \mathrm{~Pb}^{82+} 2015 \text { quench test }}{\left(\beta^{*}=80 \mathrm{~cm}\right)}$} \\
\hline & & & & & \\
\hline & & & Half gap $[\sigma]$ & Half gap $[\sigma]$ & Half gap $[\sigma]$ \\
\hline H ТCP & B1 & 7 & 5 & $5.5(\mathrm{~L})-5.0(\mathrm{R})$ & 5.5 \\
\hline V TCP & B1 & 7 & 5 & 5 & 5.5 \\
\hline $\mathrm{H} / \mathrm{V}$ TCPs & B2 & 7 & 5 & 5 & 5.5 \\
\hline TCSGs/TCLAs & $\mathrm{B} 1 / 2$ & 7 & $6.5 / 10$ & $6.5 / 10$ & $8 / 14$ \\
\hline TCP/TCSGs/TCLAs & $\mathrm{B} 1 / 2$ & 3 & $15 / 18 / 20$ & $15 / 18 / 20$ & $15 / 18 / 20$ \\
\hline H TCTs & B1 & $1 / 2 / 5$ & $15 / 15 / 15$ & $11 / 9 / 9$ & $37 / 37 / 37$ \\
\hline H TCTs & B2 & $1 / 2 / 5$ & $15 / 15 / 15$ & $9 / 9 / 9$ & $37 / 37 / 37$ \\
\hline V TCTs & $\mathrm{B} 1 / 2$ & $1 / 2 / 5$ & $15 / 15 / 15$ & $9 / 9 / 9$ & $37 / 37 / 37$ \\
\hline TCTs & $\mathrm{B} 1 / 2$ & 8 & 15 & 15 & 37 \\
\hline TCDQ/TCSP & B1 & 6 & $7.4 / 7.4$ & $7.4 / 7.4$ & $9.1 / 9.1$ \\
\hline TCDQ/TCSP & B2 & 6 & $7.4 / 7.4$ & 7.4/7.4(L)-11.2(R) & $9.1 / 9.1$ \\
\hline TCL 4/5/6 & $\mathrm{B} 1 / 2$ & $1 / 5$ & out/out/out & $15 / 15 /$ out & out/out/out \\
\hline
\end{tabular}

account their corresponding relativistic factor, $\gamma_{I}$, such that the following relation is satisfied:

$$
\frac{\epsilon_{N}^{P}}{\gamma_{P}}=\frac{\epsilon_{N}^{I}}{\gamma_{I}} .
$$

The normalized emittance value calculated using Eq. (2) for ${ }^{208} \mathrm{~Pb}^{82+}$ is shown in Table $\mathrm{I}$ and used to generate the corresponding beams for the simulations presented in Sec. V.

\section{HEAVY-ION COLLIMATION SIMULATIONS}

Heavy-ion collimation simulations must include both a high-precision particle tracking and (repetition) a good modeling of the different nuclear interaction processes of heavy ions with the collimator material, in particular electromagnetic dissociation and nuclear fragmentation, as well as an accurate tracking of the nuclear beams and out-scattered, off-rigidity fragments through the magnetic lattice.

A first software tool, ICOSIM [36] was developed in 2004. The comparison of loss maps simulated with ICOSIM and LHC BLM measurements showed that the approximated physics models and optical tracking used in this software are adequate to identify some collimation issues, but not detailed enough to model accurately the collimation of heavy ions in the LHC $[10,14]$.
In this article, we present a new simulation tool that on one hand can accurately track any heavy-ion species, and on the other hand simulates the ion-matter interactions for all tracked particles in all collimators. To achieve this, the particle tracking is done by SIXTRACK, which provides a six-dimensional tracking of relativistic beams in highenergy synchrotrons over many turns based on symplectic tracking maps. A more generic approach of computing the heavy-ion trajectories was developed by including in SIXTRACK new maps for the tracking of multi-isotopic beams, as described in the Appendix A.

The ion-matter interactions are handled by the Monte Carlo code FLUKA [37] that has the most up-todate heavy-ion physics [38]. FLUKA is used to simulate particle transport and the particle-matter interactions in a user-defined 3D geometry. Full online coupling between SIXTRACK and FLUKA was used [25,26]. This is described further in Sec. III A.

\section{A. Coupling between SixTrack and FLUKA}

The existing framework for coupling SIXTRACK and FLUKA $[25,26]$ for protons was expanded to incorporate multi-isotopic tracking [14]. The two simulation codes are run in parallel and the exchange of particles between them 


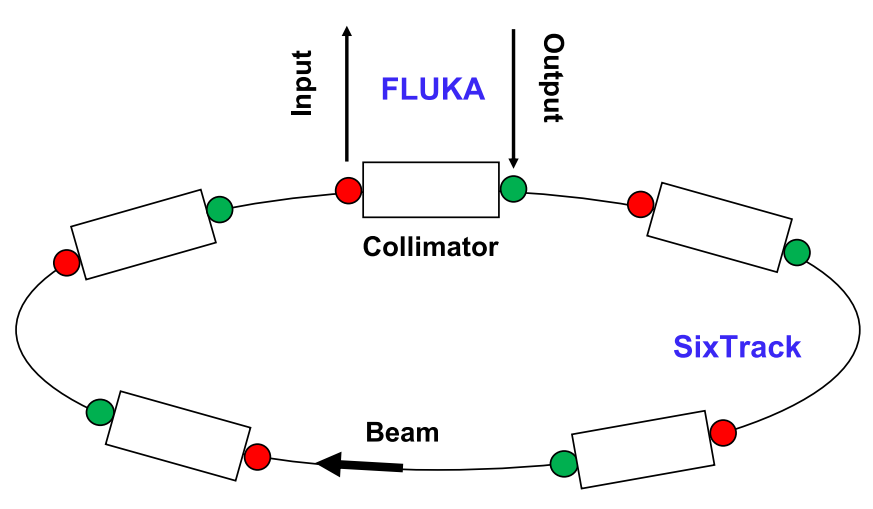

FIG. 4. SiXTrACK-FLUKA coupling principle.

is done through a network port and a C library to handle the communication between the codes. This significantly shortens the required simulation time compared to a reinitialization of each code after each particle exchange. The basic principle is illustrated in Fig. 4. At the start of every collimator there is an extraction marker (red dots in Fig. 4), and at this position the particle coordinates are sent to FLUKA where the interaction with the collimator is simulated. The implementation is done in such a way that the number of particles sent back to SIXTRACK from FLUKA can be both larger or smaller than the number of incoming particles, in order to cover for cases where ion fragments (or other secondary particles, if requested) are created or absorbed. When all input ions have been tracked through the collimator, the output distribution of particles at the end of the collimator is sent back to SIXTRACK and reinjected into the lattice at the dedicated injection markers (green dots in Fig. 4) from which the tracking is continued in SixTrack. The markers are set at the beginning and at the end of each collimator tank.

The user setup for the FLUKA simulation part is done through the generation of a standard FLUKA input file where the 3D geometry of each collimator or user-defined insertion device is defined. In this file the user can adjust the physics settings for the simulations, such as energy cuts and transport thresholds to be applied, or switch off unnecessary physical processes to optimize the computational time. The particle types sent back from FLUKA to SixTrack are also defined by the user. Typically, for heavy-ion collimation studies only nuclear fragments are considered because the fraction of energy carried by other particles is very small (see for more detail Sec. IV).

FLUKA handles only the losses at the collimators. In order to record the losses that happen in other locations around the ring an online aperture check module was developed $[25,26]$ and added to the framework. This module checks the trajectories of all tracked particles against an aperture model with a default longitudinal precision value of $10 \mathrm{~cm}$, which can be set to any other desired value by the user. The tracking stops as soon as the aperture is crossed and the impact point is recorded as well as the ion type and energy carried.

\section{SIMULATION SETUP AND ANALYSIS}

We discuss two different types of simulations in this article: betatron beam halo collimation cleaning, and BDF scenarios.

For halo cleaning studies, the number of simulated halo particles is about $3-6 \times 10^{6}$ initial heavy ions depending on the case. Our simulations have been performed with a monochromatic beam since the energy spread, typically about $10^{-4}$, is negligible compared to typical effective energy offsets resulting from nuclear fragmentation in the primary collimator. In our scenario the ${ }^{208} \mathrm{~Pb}^{82+}$ ion fragment with the smallest change of magnetic rigidity will already have an effective energy offset of about $10^{-3}$. In the noncollimation transverse plane a Gaussian distribution in the range $0-3 \sigma$ is generated, while in the collimation plane a beam halo distribution matching the phase space ellipse at the primary collimator is generated following the method outlined in [6]. In addition, only the heavy ions with a given impact parameter, $b$, defined as the distance between the heavy ions impacting the collimator and the surface of the collimator are selected. This means that the halo particles hit the collimator already on the first turn, and the physical mechanisms for the slow diffusion to larger amplitudes (e.g., electron cloud, beam-beam interactions, and intrabeam scattering to name a few) are not simulated, as this would require too much computing power and it is not relevant to evaluate the collimation system cleaning efficiency.

The actual value of $b$ in the LHC depends on the beam loss process and it is not well known and may even vary between runs. Simulations were performed with different impact parameters for the colliding $2018{ }^{208} \mathrm{~Pb}^{82+}$ optics for both beams and both planes. As an example, Fig. 5 shows

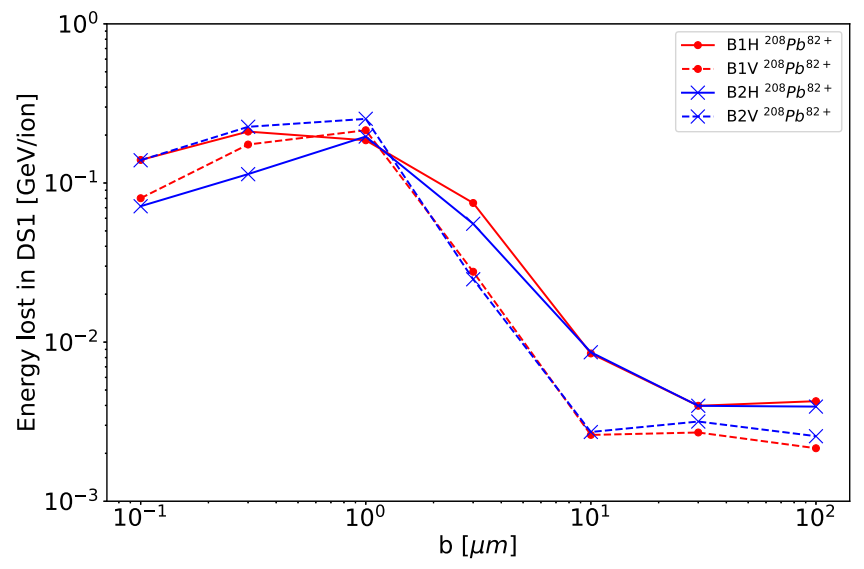

FIG. 5. Energy lost in the DS1 cluster as a function of $b$ for $6.37 \mathrm{Z} \mathrm{TeV}$ horizontal and vertical ${ }^{208} \mathrm{~Pb}^{82+}$ ion beams using the 2018 colliding optics. 

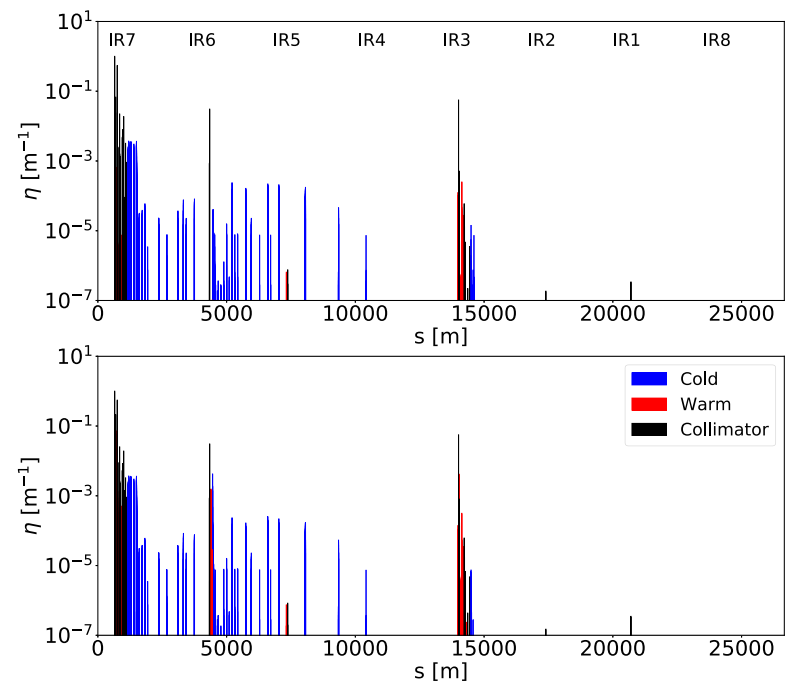

(a)

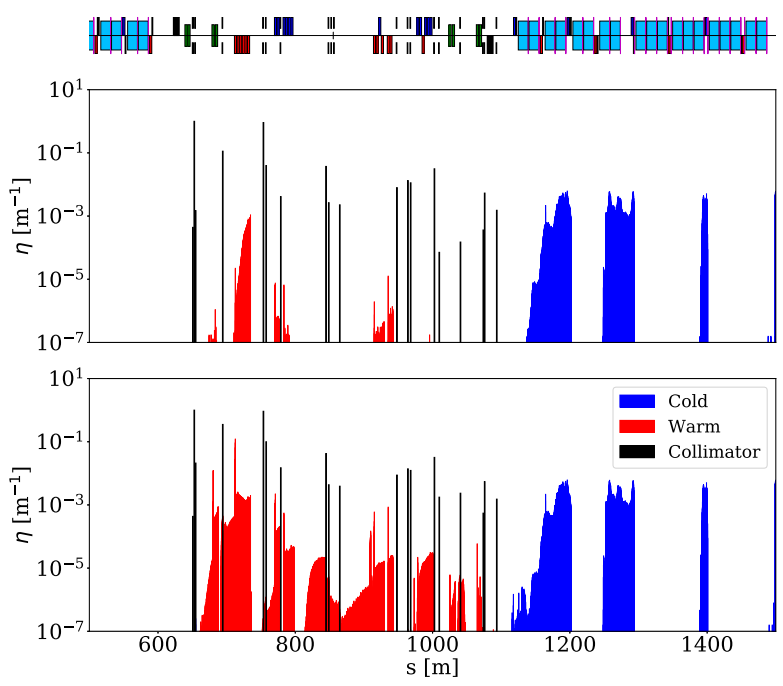

(b)

FIG. 6. B2 horizontal full ring (left) and IR7 zoom (right) loss map for $2018{ }^{208} \mathrm{~Pb}^{82+}$ ion colliding optics without (top) and with (bottom) the tracking of the protons generated at collimators.

the energy lost in the IR7 DS1 (defined between $s=$ $1150-1210 \mathrm{~m}$ in Fig. 3) as a function of the impact parameter. The observed losses in this cluster are higher for lower impact parameters with a maximum at about $1 \mu \mathrm{m}$, with the reason being that at larger impact parameters, the distance traversed inside the collimator material increases due to the angle of the beam envelope. This gives a higher probability for multiple interactions from which it is very unlikely that a heavy fragment can survive with a magnetic rigidity close enough to the main beam so that it can reach the DS. A similar behavior is observed on the losses in DS2. From this study we could conclude that for $b=1 \mu \mathrm{m}$, the energy lost in DS1 is maximized for all cases studied. Because of that, all simulations presented in the following have been performed with $b=1 \mu \mathrm{m}$, in order to stay on the pessimistic side. More detailed studies can be found in [14]. This is therefore a conservative approach for this study and for predictions of performance for future configurations.

For the purpose of the cleaning studies performed with the SiXTRACK-FLUKA coupling, which are different to local energy deposition studies, no magnetic rigidity cut is applied on the produced fragments, while a particle transport threshold cut of $1 \mathrm{TeV}$ per nucleon is considered. The particles with an energy lower than the $1 \mathrm{TeV}$ per nucleon threshold are assumed to be lost at the collimator since a $15 \%$ or lower effective energy variation is required in order to reach the cold magnets in the DS section. Particles below $1 \mathrm{TeV}$ per nucleon will already have an effective energy variation significantly higher than $15 \%$. In addition, the MULSOPT FLUKA command was activated in order to take into account the single scattering process. This process can have a small impact on the cleaning efficiency since, together with other processes, such as ionization energy loss, determines for particles that have not a pointlike interaction and survive a given turn, the impact parameter in the following turn, thus the probability of being lost.

The energy lost at the collimators can be calculated as the difference between the energy of all incoming particles, passed to FLUKA for the interaction, and the surviving particles sent back to the tracking. However, in this case the collimator losses are overestimated, since some energy is carried by particles that exit the collimator, but are not sent back to SiXTRACK. In order to correctly estimate the energy deposited in the collimators, the energy carried by these particles is saved in a file at the exit marker of the collimator. Then, this information is used in the postprocessing to correct the losses at the collimators. The two major contributing particle types are neutrons and protons.

To illustrate this and understand the impact of including or not the tracking of protons generated at the collimators, simulations have been performed with an initial population of $3 \times 10^{6}{ }^{208} \mathrm{~Pb}^{82+}$ ions and the 2018 collision optics. The simulations presented in this paper have been performed with the FLUKA2011.x.8 and SIXTRACK v5 versions.

From the simulation results, giving the loss positions around the ring, the loss maps are constructed calculating the cleaning inefficiency, $\eta(s)$, using Eq. (1). For losses in warm and cold elements, we use $\Delta s=10 \mathrm{~cm}$, corresponding to the chosen resolution of the online aperture check algorithm, while for collimators it corresponds to the collimator active length of $0.6 \mathrm{~m}$ for TCPs, $1 \mathrm{~m}$ for TCSPs, TCLAs, TCTs, TCLs and TCSPs; and $9 \mathrm{~m}$ for the TCDQ.

In Fig. 6, the full ring (left) and IR7 zoom (right) horizontal loss maps for B2 $2018{ }^{208} \mathrm{~Pb}^{82+}$ collision optics are shown without (top) and with (bottom) the tracking of the protons. Small variations in the losses at the first secondary collimators in IR7 [see Fig. 6(b)] are observed as 
well as more warm losses (red) in IR7, IR6 and IR3 due to the generation of protons in the collimators. In the IR7 TCP made of CFC about 14 protons are produced per impacting ion. These protons are lost close to the origin collimator in warm elements as can be seen in IR7 due to the magnetic rigidity difference with respect to the reference heavy ion of more than $99 \%$. The same happens to the protons originated at the TCSP and at the TCP in IR6 and IR3, respectively. The magnitude of the observed differences in collimators and cold losses is not significant when the simulation and beam measurements are benchmarked, as shown in Sec. V. Note that despite the correction for proton and neutron energies, the result is still not the real energy deposited in the collimator since the energy can escape in the form of electromagnetic and hadronic showers (e.g., pions, kaons, electrons, etc.), which are not simulated. In the following simulations presented in this paper, particles lighter than deuterium are not sent back to SIXTRACK as it is expected that losses are mainly local in IR7 while the simulation and postprocessing time is considerably increased.

The SiXTRACK-FLUKA coupling framework can also be used to perform simulations of beam dump failure (BDF) scenarios. For these simulations a special SixTRACK module $[39,40]$ is used to change dynamically the field of the extraction kicker magnets (MKDs) during the simulation, using realistic time-dependent kicks based on measured wave forms [41]. As in Ref. [30], where similar studies were performed for proton beams, we simulate several consecutive Gaussian bunches centered on the nominal closed orbit, each encountering different MKD strengths. For the results presented in this paper a $75 \mathrm{~ns}$ bunch spacing is considered; this implies that 44 bunches are kicked during the rise of the MKD fields. The first bunches receive small kicks and pass through the whole ring, later bunches receive large kicks and hit the dump protection collimator or are extracted from the machine while the intermediate bunches are the ones that risk hitting the machine aperture and sensitive collimators. Because of that, only a small range of intermediate bunches (between 5-25) are simulated using a separate three-turn simulation for each bunch. In the first turn, no MKD kick is implemented. In the second turn the simulated bunch is affected by the intermediate MKD kicks, different for each bunch. In the third turn the maximum MKD kick value is applied and the particles remaining on this turn are lost in the extraction point. Note that only the ring losses are of interest in this study and not the losses in the extraction line.

Different BDF modes can occur [42]: the single module prefire (SMPF), in which one MKD module spontaneously fires first and the remaining MKDs are then automatically triggered after a short delay, and the ABDF mode in which all MKDs fire simultaneously but at the wrong time when the beam is passing. This last mode is the one measured in the machine and for which comparison studies with simulations have been performed and presented in Sec. V.

\section{APPLICATIONS AND COMPARISON OF SIMULATIONS WITH MEASUREMENTS}

In this section, we show examples of applications of our simulation setup to the LHC operation during the 2015 and $2018 \mathrm{~Pb}-\mathrm{Pb}$ runs. The simulations demonstrated to be a very good guide to understand the origin of the losses in various complex configurations and to optimize the collimator settings and formulate mitigation strategies. Simulated and measured loss maps for the betatron cleaning and $\mathrm{ABDF}$ scenarios are compared and the agreement is discussed. It should be noted that quantitative discrepancies up to at least a factor of 10 can be observed in the comparison of simulated and measured loss maps [6]. This is because the simulated loss maps show the sum of the energy of the lost particles impacting on the aperture around the ring, while the measured losses are taken from the BLM signals. Since the BLMs measure the secondary particles created in the showers, the ratio between local losses and BLM signal can vary significantly between different locations, depending on the materials and local geometry as well as the impact distribution.

A more quantitative comparison between simulation and measurements can be obtained with energy deposition simulations of the local showers at critical loss locations (e.g., with FLUKA), including the local geometry and the BLM and hence better representing the BLM response. However, this is only possible for a few locations and not for the full ring due to the required resources to prepare detailed local geometries and to the computational time. In order to illustrate the improved quantitative agreement when performing detailed energy deposition simulations, IR7 results obtained with the full simulation chain (i.e., tracking and showering) for the $2015 \mathrm{~Pb}-\mathrm{Pb}$ collimation quench test are presented in Sec. V B. For the purpose of this paper, it will be shown that the comparison based on loss maps is adequate.

\section{A. $2018{ }^{208} \mathrm{~Pb}^{82+}$ ion run}

During the commissioning of the $2018 \mathrm{~Pb}-\mathrm{Pb}$ run, losses were observed at the horizontal TCT (TCTPH) in IR1 in the physics configuration (see Table II) that could potentially cause high background or beam dumps [43]. An effort was made to decrease these losses and the SixTrack-FLUKA simulations were then used to understand the loss sources and to propose mitigation measures.

The simulations showed that the losses at the TCTPH consisted mainly of ${ }^{207} \mathrm{~Pb}^{82+}$ ions, which were created inside the left TCP jaw (while moving in the beam direction) and then bypassed all other collimators. The ${ }^{207} \mathrm{~Pb}^{82+}$ ion is the fragment with the smallest rigidity offset and it stays within the acceptance of the arcs, so it can travel 


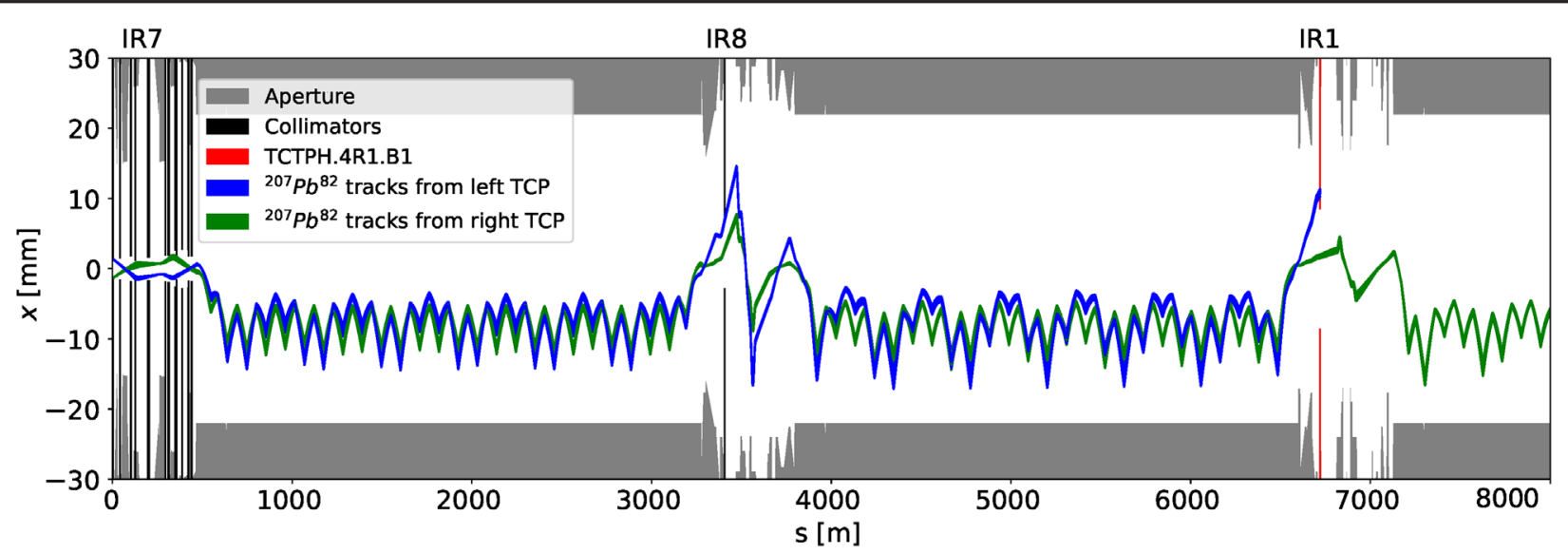

FIG. 7. ${ }^{207} \mathrm{~Pb}^{82+}$ ion orbit from the TCP to IR1 together with the aperture model and collimators with TCTPH.4L1.B1 at 9 $\sigma$ half gap highlighted in red for the $2018{ }^{208} \mathrm{~Pb}^{82+}$ ion run physics optics.

far along the machine circumference. In Fig. 7 the tracks of ${ }^{207} \mathrm{~Pb}^{82+}$ ions originated on the left (blue) and right (green) TCP jaw are shown from the TCP to IR1 together with the aperture model and collimators with the TCTPH indicated in red. Fragments emerging from the two TCP jaws are lost at different locations, because the betatron motion is either amplifying or compensating the dispersive offsets depending on the starting betatron phase. This was an important result from simulations that was tested and applied in LHC operation. Note that this could not be found out only through measurements as it is not possible to test many different collimator configurations during the commissioning phase due to time constraints. Therefore, accurate simulation results are crucial to anticipate possible issues and elaborate loss mitigation strategies.

We therefore proposed to decrease the TCTPH losses by either slightly retracting the ТCTPH jaws, which was possible since there was significant margin to the triplet aperture, or by concentrating the primary losses on the right TCP jaw only. The latter option could be achieved by retracting the left TCP jaw by a small amount. Figure 8 shows a comparison of the simulated (top) and measured (bottom) horizontal loss maps for B1 with both TCP jaws closed to $5 \sigma$ [Fig. 8(a)], with only the right TCP jaw closed to $5 \sigma$ and the left jaw opened to $36 \sigma$ [Fig. 8(b)] and with only the left TCP jaw closed to $5 \sigma$ and the right jaw opened to $36 \sigma$ [Fig. 8(c)].

A very good qualitative agreement between simulations and measurements is observed on the losses at the collimators. The losses at the TCTPH in IR1 (at $s \approx 7500 \mathrm{~m}$ ) are reduced by more than 3 orders of magnitude when the left TCP jaw is opened in both simulated and measured loss maps. In addition, when the left TCP jaw is opened, no losses are observed at the TCTPH in IR5 and at the TCSP in
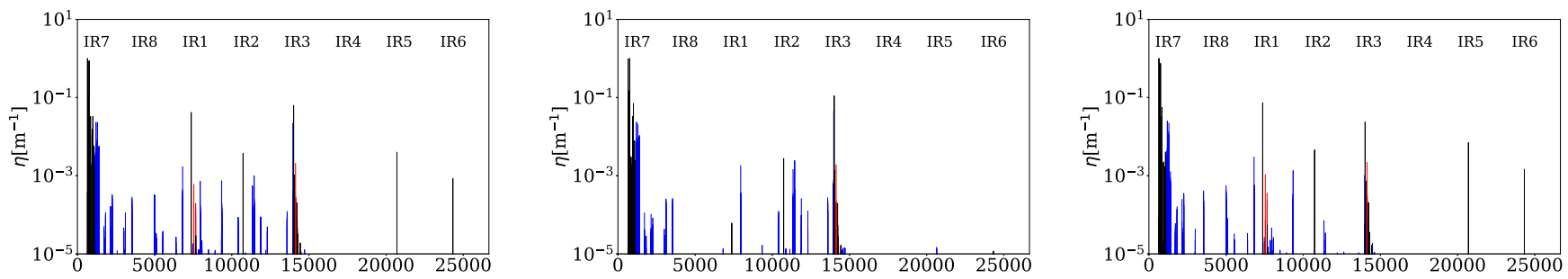

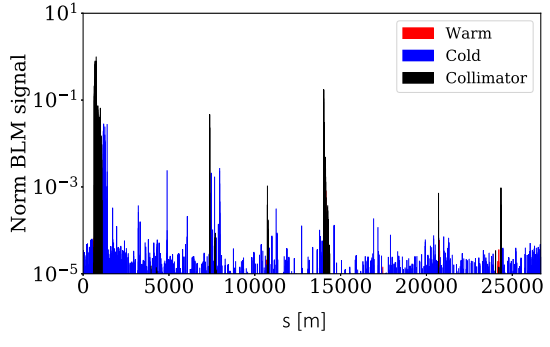

(a)

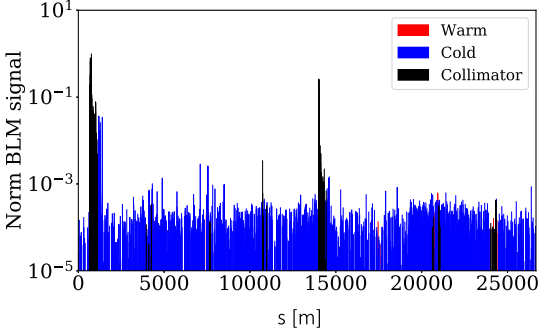

(b)

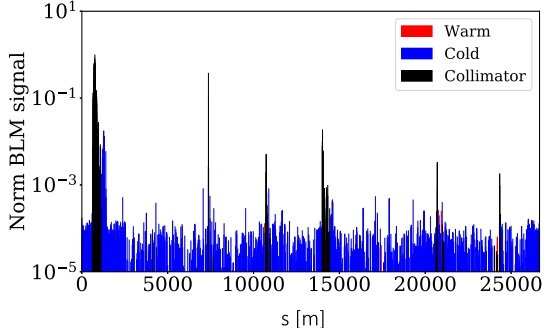

(c)

FIG. 8. Horizontal B1 simulated (top) and measured (bottom) loss map with both TCP jaws closed to $5 \sigma$ (a), with only the right TCP jaw closed to $5 \sigma$ (b) and with only the left TCP jaw closed to $5 \sigma$ (c). 


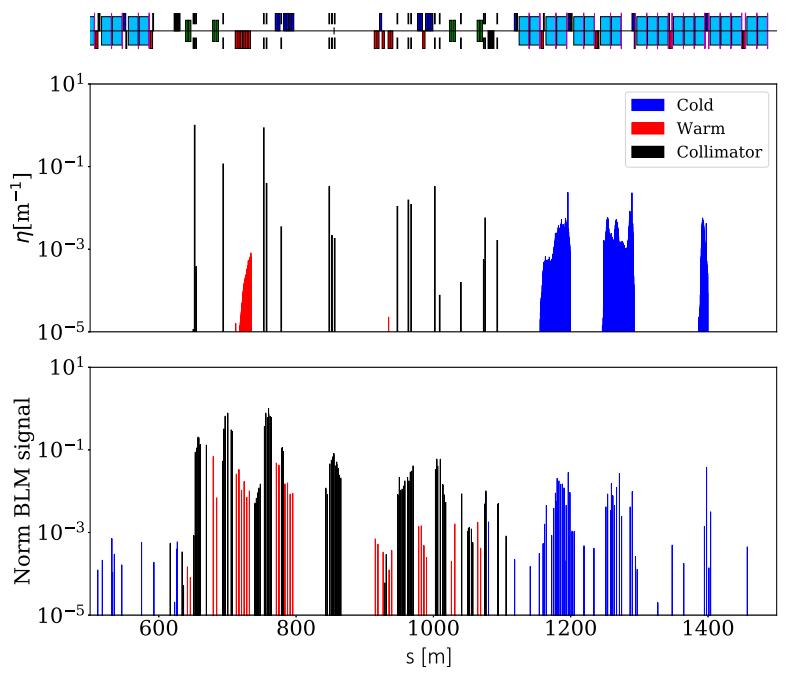

FIG. 9. IR7 horizontal B1 simulated (top) and measured (bottom) loss map for both TCP jaws at $5 \sigma$.

IR6 while the losses in the TCP in IR3 slightly increase, in both simulations and measurements. Quantitatively, discrepancies up to 1 order of magnitude are observed, for example on the level of losses in the TCTPH in IR1 when only the left TCP jaw is considered. These levels of discrepancies are in the order of what is expected due to the difference in BLM response as discussed in the introduction of this section. In addition, note that no orbit and machine errors are taken into account in the simulations which could also contribute to these observations.

Concerning cold losses, spikes between IR7 and IR1 when both TCP jaws are in place, as seen in Fig. 8(a), are observed in both simulations and measurements. These losses are mainly coming from the left TCP jaw. The longitudinal location of these spikes is in some cases shifted or even absent in one of the two (measured or simulated). These differences could be explained by aperture or orbit displacements in the real machine that are not reproduced in simulations. However, the location with the highest amount of cold losses is very well reproduced as can be seen in Fig. 9, where the simulated (top) and measured (bottom) IR7 loss maps are shown. The losses at the three clusters, named in Sec. II as DS1, DS2, and DS3, where the locally generated dispersion function from the TCP is beating with amplitudes between 0-2.1 m, are very well reproduced by the simulations. Concerning the losses at the collimators in IR7, quite good agreement can be observed on the hierarchy, however in measurements the signals are higher at the secondary collimators than at the primary collimators, but not in simulations. This difference with respect to the simulations could be due to the fact that IR7 BLMs intercept showers also from losses further upstream and to the BLM response, accounted for by a second step of additional energy deposition simulations discussed in Sec. V B where the quantitative agreement is significantly improved.

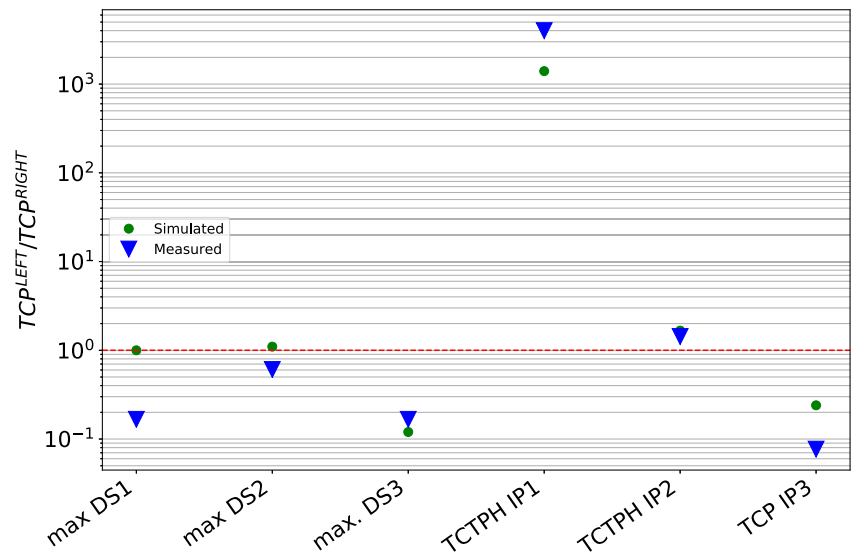

FIG. 10. Simulated (green) and measured (blue) ratio of losses generated by the left and right TCP jaws at $5 \sigma$ at different locations. Note that only collimators with BLM signals above the noise level are considered.

To illustrate the differences between the loss map generated by only the left TCP jaw and only the right TCP jaw at $5 \sigma$, Fig. 10 shows the ratio of losses obtained in simulations (green) and measurements (blue) between the two loss maps at different locations. In general, a very good agreement is observed with the trend in all depicted locations between simulations and measurements, in particular since the BLM response is not included. Note that the data cover several orders of magnitude.

Simulations have also been performed with the flattop machine configuration with the optics, beam, and collimator settings summarized in Tables I and II. The comparison of simulated (top) and measured (bottom) loss maps for B1 in the horizontal plane is shown in Fig. 11.
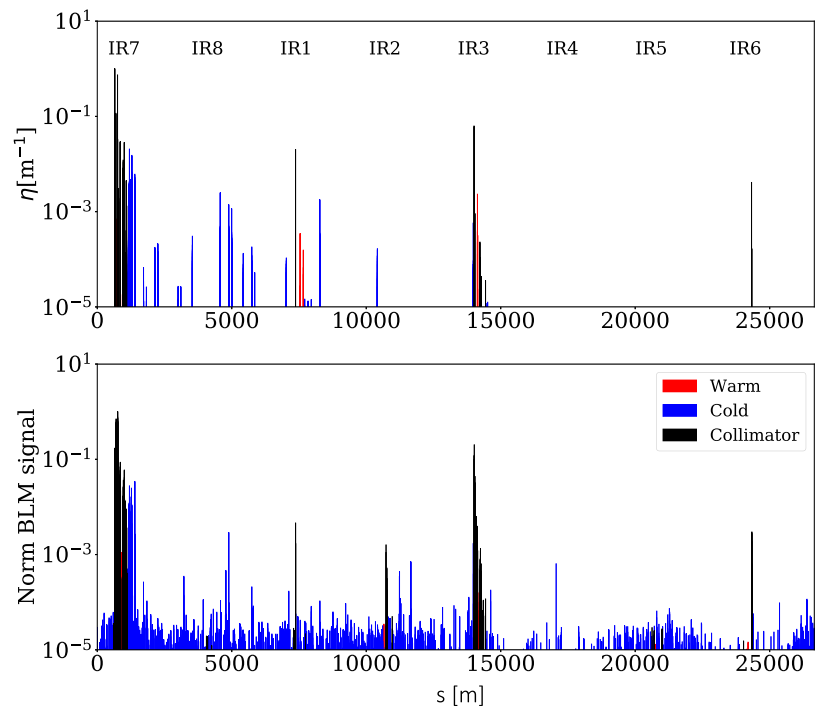

FIG. 11. Simulated (top) and measured (bottom) FT B1 horizontal ${ }^{208} \mathrm{~Pb}^{82+}$ ion beam loss map. 

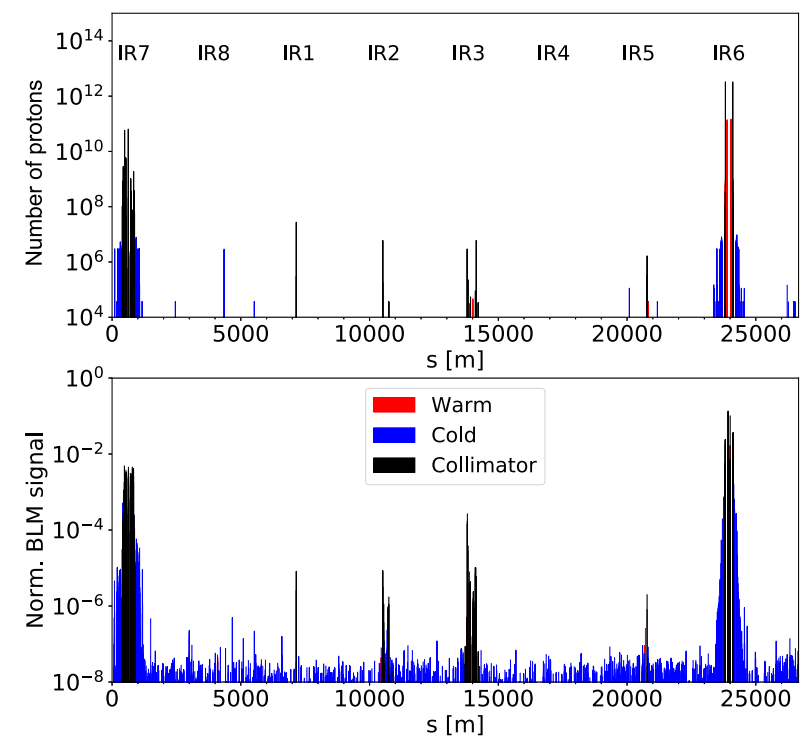

FIG. 12. Simulated (top) and measured (bottom) loss map for an ABDF test with colliding beams during the $2018{ }^{208} \mathrm{~Pb}^{82+}$ ion run commissioning.

A quite good qualitative agreement can be observed in the losses at the collimators except for the losses on the TCT in IR2 that are present in measurements, but not in simulations. This could potentially be explained by various imperfections, but a more detailed study would be required to verify this hypothesis. Cold spikes between IR7 and IR1 are observed in both simulations and measurements and the highest losses in the DS of IR7 are again well reproduced.

In comparison to the simulations performed with the small- $\beta^{*}$ optics, less losses are observed at the TCTs in IR1, IR2, and IR5 while in IR7 no significant changes are observed, as expected, since in IR7 the optics and collimator settings are the same for both optics.

ABDF failure measurements performed during the 2018 ${ }^{208} \mathrm{~Pb}^{82+}$ ion commissioning have also been used to evaluate the SIXTRACK-FLUKA coupling framework. In measurements only an event similar to the ABDF failures can be caused intentionally. As explained in [30] the SMPF is the most critical failure mode, since more bunches are kicked at the most critical amplitudes. Because of that, in simulations also the SMPF has to be considered to evaluate the most pessimistic failure scenario in terms of beam losses around the machine and evaluate the amount of losses on the most sensitive components. The SixTrackFLUKA coupling was used to perform simulations of such failure scenarios, for the first time with ion beams.

As an example, in Fig. 12 the simulated (top) and measured (bottom) loss maps, resulting from a 2018 ABDF test performed with ${ }^{208} \mathrm{~Pb}^{82+}$ ions and collision optics, is shown. It should be noted that in both plots, data from the two beams are superimposed, since the experimental procedure is performed for the two beams
TABLE III. $2018{ }^{208} \mathrm{~Pb}^{82+}$ ion run optics $\Delta \mu^{\text {MKD-TCT }}$ summary for $\mathrm{B} 1$ and $\mathrm{B} 2$.

\begin{tabular}{lcc}
\hline \hline $\mathrm{IR}$ & $\Delta \mu_{\mathrm{B} 1}^{\mathrm{MKD}-\mathrm{TCTPH}}$ & $\Delta \mu_{\mathrm{B} 2}^{\mathrm{MKD}-\mathrm{TCTPH}}$ \\
\hline 1 & $176^{\circ}$ & $151^{\circ}$ \\
2 & $223^{\circ}$ & $212^{\circ}$ \\
5 & $162^{\circ}$ & $176^{\circ}$ \\
\hline \hline
\end{tabular}

simultaneously. In addition, the simulated loss map is given in number of protons for comparison with collimator damage limit calculations performed for $6.5 \mathrm{TeV}$ protons in [44]. For $\mathrm{Pb}$ ions, we look at the total impacting energy on the collimator and compare with the total impacting proton energy that would cause damage. This approximation holds since the peak power deposition for $6.37 \mathrm{Z} \mathrm{TeV}$ $\mathrm{Pb}$ ions on tungsten is driven by the hadronic shower, which is similar for protons and ions. The energy loss from ionization of the $\mathrm{Pb}$ ions is for this particular case well in the shadow of the hadronic shower. Good qualitative agreement is observed, with the main losses occurring this time in IR6, downstream of the extraction, with about 1 order of magnitude lower losses in IR7. Losses are observed also at the TCTs for B1 in IR1, for both beams in IR2, and at a lower level for B2 in IR5. Cold spikes are also observed in the arcs between IR8 and IR1 in both simulations and measurements. As discussed at the beginning of this section, the observed shifts in the longitudinal axis could be due to orbit and machine imperfections.

Serious damage can be caused to the TCTs if the primary and focused beam is intercepted (see [44] for the case of protons). In order to ensure the protection of these collimators for the operation at small $\beta^{*}$ when tight TCTs settings are required, the fractional phase advance between the MKDs and these collimators has to be within $30^{\circ}$ from $0^{\circ}$ or $180^{\circ}$ with proton beams [30]. In Table III a summary of the phase advance between MKDs and TCTs $\left(\Delta \mu^{\mathrm{MKD}-\mathrm{TCT}}\right)$ for both beams in the $2018^{208} \mathrm{~Pb}^{82+}$ optics is presented. As can be seen, the TCTs in IP2 do not satisfy the requirements. Because of that, it was important to quantify the expected impacts for ${ }^{208} \mathrm{~Pb}^{82+}$ ion beams, in order to conclude whether the phase advance could be accepted. However, the ${ }^{208} \mathrm{~Pb}^{82+}$ ion beams used at the LHC have a smaller bunch intensity and larger bunch spacing than the proton beams and the phase advance tolerances in such failure scenarios can be larger than for protons.

A sensitivity study was done, simulating the accidental losses as a function of the TCT half gap. This allows establishing the proper margins when defining the collimator settings, accounting for possible orbit errors during operation as in [29].

In Fig. 13 the results of these simulations are presented in the form of scaled energy lost at the TCTs in the different IRs as a function of the TCT half gap for B1 (top) and B2 

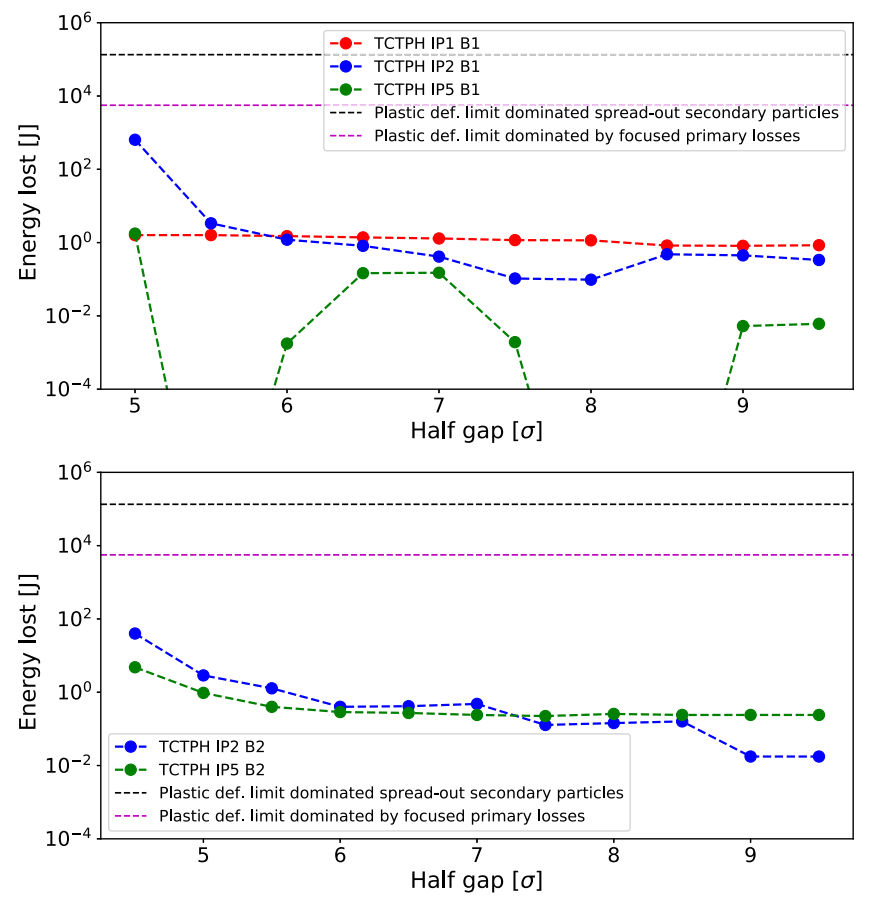

FIG. 13. Losses at the TCTs at the higher luminosity experiments from simulation results of a SMPF mode scenario as a function of the TCTs half aperture for B1 (top) and B2 (bottom). Note that the discontinuity observed on the top plot green line is due to statistic limitations and indicates nearly no losses at this collimator. The energy lost by one primary ${ }^{208} \mathrm{~Pb}^{82+}$ ion of $6.37 \mathrm{Z}$ $\mathrm{TeV}$ in the simulations after the applied normalization and scaling as explained in the text, corresponds to $1.5 \times 10^{-1} \mathrm{~J}$ in this plot. For B2 no losses are observed in the TCT in IR1.

(bottom), with all other collimators set according to the 2018 physics configuration in Table II. The losses have been calculated as the sum over all simulated bunches normalized to the total energy simulated and scaled to the real beam intensity in the machine for the number of bunches simulated. The resulting energy has been expressed in Joules.

In Fig. 13, the losses at the tungsten TCTs are compared with the estimated damage limits for protons [44] obtained from detailed energy deposition and thermomechanical studies. The proton damage limit can be used in fairly good approximation, since the hadronic shower is very similar to the ${ }^{208} \mathrm{~Pb}^{82+}$ ions. While ionization energy loss scales with $Z^{2}$ and is thus much higher for nuclear beams, it means it can only cause the peak energy deposition at very small spot sizes. The purple dashed line indicates the plastic deformation limit for primary and focused beam losses, while the dashed black line corresponds to the plastic deformation limit for secondary spread-out beam.

A flat dependence of the losses on the TCT setting can be observed for all the TCTs down to a minimum half gap of $5.5 \sigma$. Only for the horizontal TCT in IP2 we observe a steep rise when closing from $5.5 \sigma$ to $5 \sigma$. This means that up to this point all intercepted particles are from secondary spread-out beam, out-scattered at large amplitudes by the dump protection, as in Ref. [30], and the values are well below the damage limit from secondary beam halo. Notice that the discontinuity observed in Fig. 13 for the TCTPH in IR5 (in green) could be explained by statistical limitations since a different initial beam distribution is generated for each bunch with only $12 \times 10^{4}{ }^{208} \mathrm{~Pb}^{82+}$ ions. This indicates nearly no losses as the energy lost in the simulations by one primary ${ }^{208} \mathrm{~Pb}^{82+}$ ion of $6.37 \mathrm{Z} \mathrm{TeV}$ corresponds to $9.3 \times 10^{8} \mathrm{GeV}$ in this plot. As can be seen in Fig. 13 (bottom) for B2 losses are observed on the TCT in IR1.

From these studies we could conclude that the losses at the TCTs were well below the plastic deformation limits (indicated in black and purple in Fig. 13) with an operational margin of $3.5 \sigma$ between the operational TCT setting and the settings at which there is a risk for damage for the $2018{ }^{208} \mathrm{~Pb}^{82+}$ ion collision optics, corresponding to the abrupt change on the slope of the curves of Fig. 13. The results show that the optics requirements, e.g., in terms of MKD-TCT phase advance, for ${ }^{208} \mathrm{~Pb}^{82+}$ ion beams are less stringent than the ones for protons in Ref. [30]. This is mainly due to the much lower bunch charge and larger bunch spacing, which both contribute to the lowering of the total impacting energy. Note that the phase advance window is larger for ${ }^{208} \mathrm{~Pb}^{82+}$ ions but not irrelevant since one typical $2018^{208} \mathrm{~Pb}^{82+}$ ion bunch of $2 \times 10^{8}$ ions carries $18.4 \mathrm{~kJ}$, which is significantly above the plastic deformation limit $(5.6 \mathrm{~kJ})$ and close to the fragment ejection limit for equivalent proton energies.

\section{B. $2015{ }^{208} \mathrm{~Pb}^{82+}$ quench test}

In a "collimation quench test," losses on the primary collimator are intentionally increased in a controlled way, causing losses in the cold DS magnets immediately downstream of the collimation insertion that could potentially cause a quench. The level of losses at which the quench occurs, or the largest losses without quench, can be compared with the estimated quench limit [45]. These measurements provide almost ideal conditions for benchmarking simulations due to the resulting high signal-tonoise ratio and the control of the loss source. In addition, further information from the quench experiment performed in 2015 such as the power deposition in the SC magnet coils could be gathered by means of combining the measured loss rates with simulations which is of great importance to evaluate possible limitations in future runs with envisaged higher intensities. Figure 14 shows the SIXTRACK-FLUKA coupling simulated (top) and measured (bottom) horizontal B2 ${ }^{208} \mathrm{~Pb}^{82+}$ ion full ring [14(a)] and IR7 zoom [14(b)] loss map for the 2015 quench test configuration, shown in Table I. Good qualitative agreement can be observed in the losses at the collimators except for the small amount of losses (about $10^{-4}$ ) observed in the 

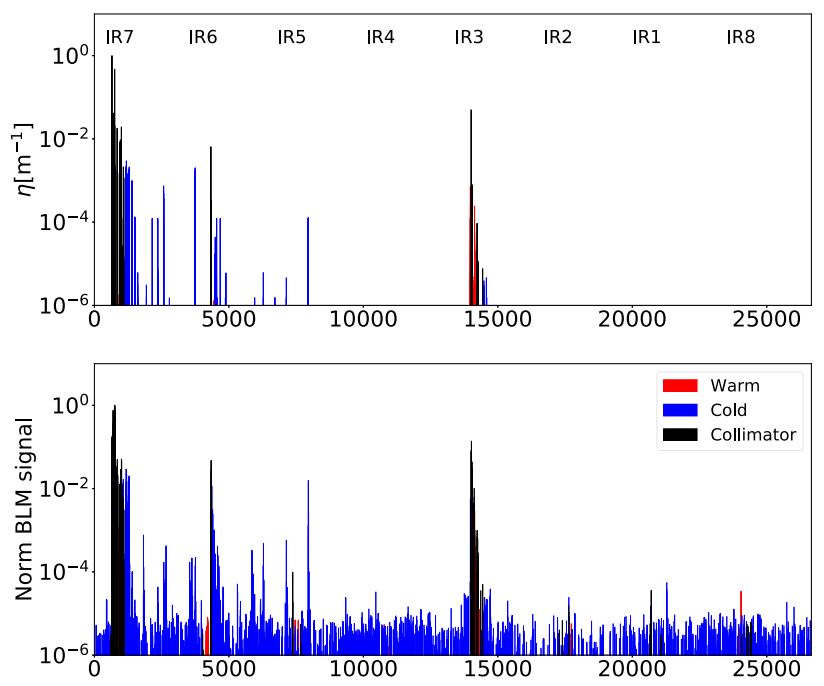

(a)

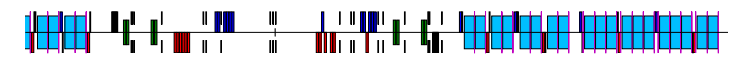
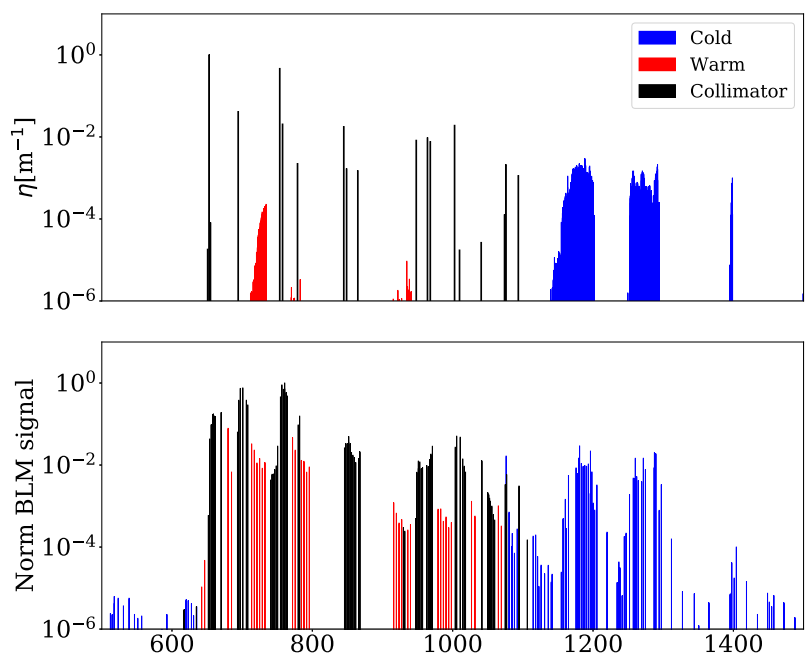

(b)

FIG. 14. Simulated (top) and measured (bottom) horizontal B2 full ring (a) and IR7 zoom (b) loss map for the $2015^{208} \mathrm{~Pb}^{82+}$ ion beam collimation quench test.

measured loss map on the TCT in IR5 that are not present in simulations. Cold spikes between IR7 and IR5 are observed in both simulations and measurements and the highest losses in the DS of IR7 are again well reproduced.

As anticipated in the introduction of this section, an improved quantitative comparison can be achieved by a two-step simulation combining tracking and detailed energy deposition studies. A similar approach was already adopted in Ref. [45] for benchmarking BLM response simulations for proton collimation losses. In a first step, the SiXTRACK-FLUKA coupling was used to generate the impact distribution of ${ }^{208} \mathrm{~Pb}^{82+}$ ions at the primary collimator in IR7. In the second step, this impact distribution at the TCP was employed as initial distribution for the FLUKA energy deposition simulation with a detailed model of the IR7 geometry and including all magnetic fields of the warm and cold magnets in the FLUKA model. In these simulations, the interaction of the particles with the primary collimator material and the subsequent propagation of residual fragments and electromagnetic and hadronic showers in the surrounding machine hardware is modeled. Secondary particles were transported until their kinetic energy fell below $1 \mathrm{MeV}$ (electrons, positrons), $100 \mathrm{keV}$ (photons, hadrons, muons) or $10^{-5} \mathrm{eV}$ (neutrons). Detailed models of the loss monitors used in the LHC (nitrogen-filled ionization chambers with a sensitive volume of about $1500 \mathrm{~cm}^{3}$ ) were included in the simulation setup to perform a quantitative comparison with the measured BLM signals. Since only a small fraction of secondary particles leak to superconducting magnets in the DS and arc, separate FLUKA shower simulations were carried out for the room temperature and the cold accelerator region. To enhance the statistical convergence in the latter case, the FLUKA simulation was split into two steps. In the first step, the transport of secondary particles and ion fragments emerging from interactions in collimators was suppressed if they could not reach the DS because of their magnetic rigidity. In this way, a representative distribution of secondary particles leaking to the cold region could be obtained since the computational time for simulating particle transport in the insertion region was significantly reduced. In the second step, the showers induced by the particles lost in cold magnets were simulated using the low transport thresholds mentioned above. More details about this simulation approach can be found in [46]. The two steps were not necessary for obtaining BLM signals in the insertion region itself since a good statistical convergence could be achieved in a single step.

Figure 15 compares simulated and experimental BLM signals in IR7, the neighboring DS, and the first arc cell. The position of the primary and secondary collimators, tungsten absorbers and magnets is indicated on the top of the graph. The BLM signals are expressed per ${ }^{208} \mathrm{~Pb}^{82+}$ ion intercepted by the IR7 collimators and eventually lost in the machine. The simulation results represent the dose scored in the active gas volume of the BLM models. The experimental BLM dose values were derived by timeintegrating BLM signals over the entire loss duration and by dividing the results by the number of ions lost from the beam. The latter was determined from beam current measurements, which provided a good estimate of betatron losses in IR7 since the contributions of other loss mechanisms were much smaller. In total, about $1.4 \times 10^{9}$ ${ }^{208} \mathrm{~Pb}^{82}+$ ions were lost during the experiment. The 


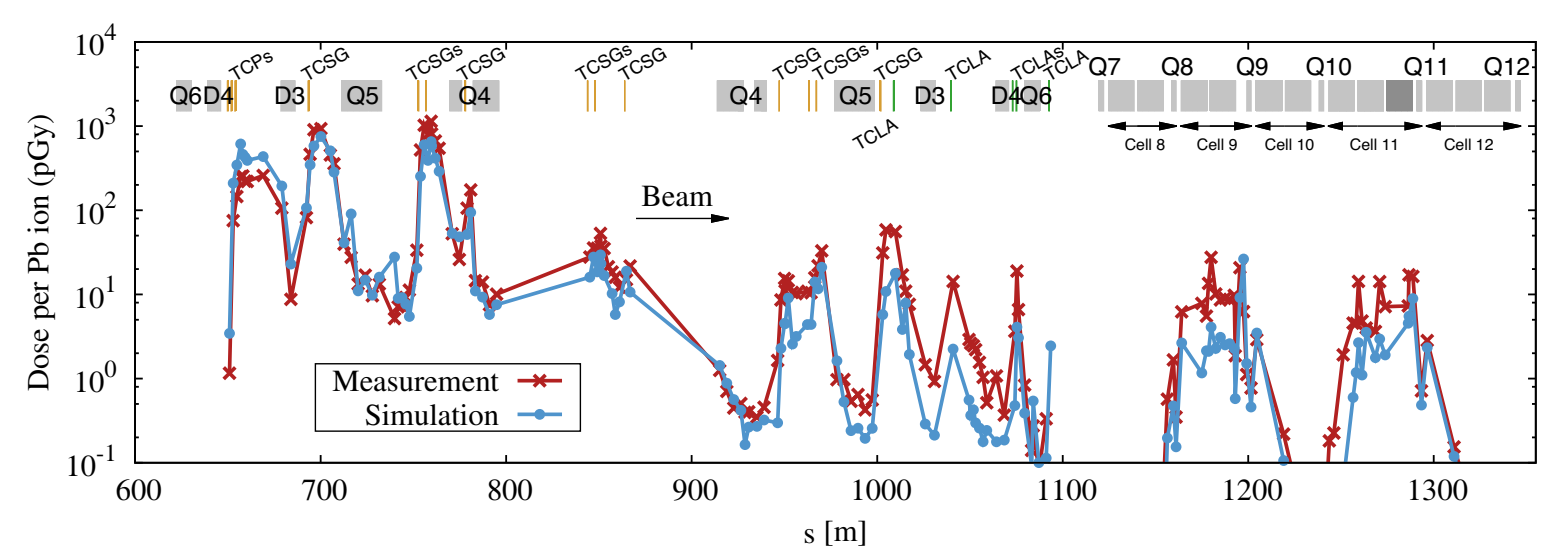

FIG. 15. Comparison of simulated and measured BLM signals in the IR7 cleaning insertion, the adjacent DS (cells 8-11) and the first arc cell (cell 12). The beam direction is from the left to the right. The signals are expressed per ${ }^{208} \mathrm{~Pb}^{82+}$ ion lost in the collimation system. The positions of primary and secondary collimators (TCPs and TCSGs), tungsten absorbers (TCLAs), dipoles (D3 and D4), and quadrupoles (Q4-Q12) are illustrated on the top. The statistical error of simulation results is generally smaller than $10 \%$ for dose values above 10 pGy but can be significantly larger for dose values below 10 pGy where a lower amount of losses occurs.

measured BLM dose values were also corrected for the noise pedestal, which was obtained from a reference period without beam just after the experiment. The noise contribution was less than $1 \%$ for the highest signals at primary and secondary collimators, and between $1 \%$ and $10 \%$ for the highest signals in the DS and arc.

In general, the simulated BLM pattern reproduces well the measured pattern, which spans several orders of magnitude in losses, over more than $600 \mathrm{~m}$. Qualitative features like elevated BLM signals downstream of collimators and the two loss clusters in the DS are well reproduced. A good quantitative agreement, better than a factor of 2-3, can be observed for most BLMs at primary and secondary collimators. The simulation systematically underestimates, however, measured signals in the second half of the insertion region and in the cold accelerator region up to the Q11. The simulated signals in the DS are on average about 4 to 5 times lower than the measured ones. This can possibly be attributed to the assumed ${ }^{208} \mathrm{~Pb}^{82+}$ impact distribution on TCPs and to machine imperfections that are not taken into account in the model. As shown in [14], the chosen maximum impact parameter of ${ }^{208} \mathrm{~Pb}^{82+}$ ions on the TCPs influences the number of fragments leaking to the DS. In the present study, the maximum impact parameter was assumed to be $2 \mu \mathrm{m}$. If the actual value was smaller, then this could have lead to a higher leakage as can be seen in Fig. 5. In addition, machine imperfections like collimator tilts can also affect the collimation inefficiency. Imperfections could also be the main reason why the leakage of single diffractive protons to the DS was underestimated by about a factor of 3 in previous proton benchmark studies [45,47]. As shown in [46], the agreement for protons notably improved if primary collimators were assumed to be tilted. Qualitatively, a similar effect is expected for the leakage of ${ }^{208} \mathrm{~Pb}^{82+}$ fragments. Despite the observed underestimation in Fig. 15 the results are nevertheless considered remarkable, given the complexity of the simulation chain and the large variation of BLM signals, which span over many orders of magnitude.

\section{HL-LHC EXPECTED PERFORMANCE}

The LHC run 1 (2010-2013) [48] and run 2 (2015-2018) [15] collimation operation was very satisfactory, with no collimation-induced quenches, however relevant upgrades are necessary to cope with the beams foreseen by the HL-LHC project [49,50], which aims at increasing the integrated proton and heavy-ion luminosity collected by the LHC experiments by a factor 10 over twelve years. To reach this goal, it is, among others, foreseen to use a higher proton-bunch population at injection of about $2.3 \times 10^{11}$ and to increase the number of bunches in the heavy-ion operation from 733 , as used in 2018 , to 1232 , thanks to a shorter $50 \mathrm{~ns}$ bunch spacing [51].

To cope with these high-intensity beams, some upgrades are planned for the collimation system with the aim of reducing the impedance contribution from the collimation system, to improve the cleaning efficiency in IR7, and to protect the experimental IRs from physics debris. The planned upgrade to improve the cleaning in IR7 is motivated mainly by predicted limitations in the HLLHC collimation performance with heavy-ion beams. This limit is more constraining for ${ }^{208} \mathrm{~Pb}^{82+}$ ion beams than for proton beams in spite of the factor 35 lower stored beam energy carried by the ${ }^{208} \mathrm{~Pb}^{82+}$ ion beams, due to the observed worsening of the IR7 cleaning performance (see Fig. 3). Based on energy-deposition studies [52], the observed heavy-ion losses in simulations in the DS downstream of IR7 (see Fig. 17 top graph) scaled up to HL-LHC intensities are above the quench limit for beam lifetime drops below the design value of 12 minutes. To overcome this limitation, one new collimator per beam, called TCLD, 


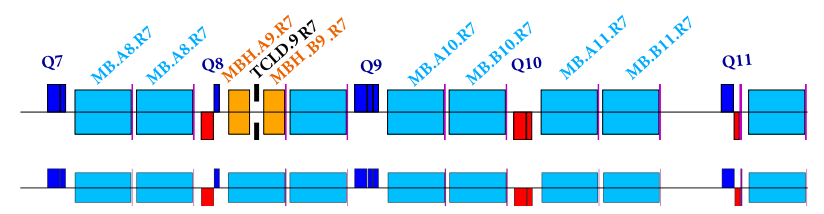

FIG. 16. Run 3 (top) and run 2 (bottom) layouts of the IR7 DS for B1, with and without the new TCLD collimators.

will be installed in cell 9 on each side of IR7, as illustrated in the layouts in Fig. 16. This collimator has an active length of $0.6 \mathrm{~m}$ and the jaws made of Inermet-180. To make room for the TCLDs, a standard main dipole will be replaced by an assembly consisting of two shorter and stronger $11 \mathrm{~T}$ dipoles [53] (in orange in Fig. 16) with the TCLD in the space in the middle.

The performance of the collimation system for the new layout has been simulated using the SIXTRACK-FLUKA coupling for ${ }^{208} \mathrm{~Pb}^{82+}$ ion beams and the HL-LHC v1.2 optics. A $7 \mathrm{Z} \mathrm{TeV}{ }^{208} \mathrm{~Pb}^{82+}$ ion beam with a normalized emittance of $1.49 \times 10^{-6} \mathrm{~m} \mathrm{rad}$ has been generated following the method described in Sec. IV. The collimator settings considered in these simulations are summarized in Table IV. Note that other collimator settings are being considered, but it is not expected that they will affect the conclusions of the studies presented in this paper.

In Figs. 17 and 18, the simulated horizontal LHC (top) and HL-LHC (bottom) loss maps are shown for B1 and B2, respectively. The full ring loss maps are presented in Figs. 17(a) and 18(a) and a zoom of IR7 is shown in Figs. 17(b) and 18(b) for B1 and B2, respectively. With the TCLD in place in IR7, the cleaning all along the machine is greatly improved, as can be seen in both Figs. 17 and 18 .
TABLE IV. HL-LHC ${ }^{208} \mathrm{~Pb}^{82+}$ ion physics collimator settings for a normalized proton emittance of $\epsilon_{N}^{P}=3.5 \mu \mathrm{m}$.

\begin{tabular}{lcc}
\hline \hline Collimator & IR & Half aperture $[\sigma]$ \\
\hline TCP/TCSG/TCLA & 7 & $6 / 7 / 10$ \\
TCP/TCSG/TCLA & 3 & $15 / 18 / 20$ \\
TCTs & $1 / 2 / 5 / 8$ & $10 / 10 / 10 / 15$ \\
TCDQ/TCSP & 6 & $9 / 9$ \\
TCL 4/5/6 & $1 / 5$ & 12 \\
TCLD & 7 & 14 \\
\hline \hline
\end{tabular}

For B1, collimator losses are localized in IR7, IR3 and at the horizontal TCTPH in IR1. High losses in the TCTPH in IR1 are expected at the same level as observed in the 2018 ${ }^{208} \mathrm{~Pb}^{82}+$ ion run. In order to reduce the losses at this collimator, as shown in Sec. V, one could in principle open the TCP left jaw or the TCTPH jaws. For B2, losses at collimators are only observed in IR7 and IR3. It should be noted especially that cold spikes all along the ring are almost fully suppressed, in particular, in IR7 where the limiting cluster losses defined in Fig. 3 as DS2 and DS3 are completely mitigated and in DS1 the losses are reduced by more than a factor 10. This is because all these suppressed losses were caused by ion fragments with a magnetic rigidity different from the main beam. These fragments are efficiently intercepted by the TCLD that is in a location with a significant dispersion. Detailed energy deposition studies have shown that the remaining cold losses occurring upstream of the TCLD are not limiting the achievable beam intensity [52]. In addition, the heat load on downstream magnets from the secondary showers from the TCLD has been shown in simulations to stay well below the quench limit [20].
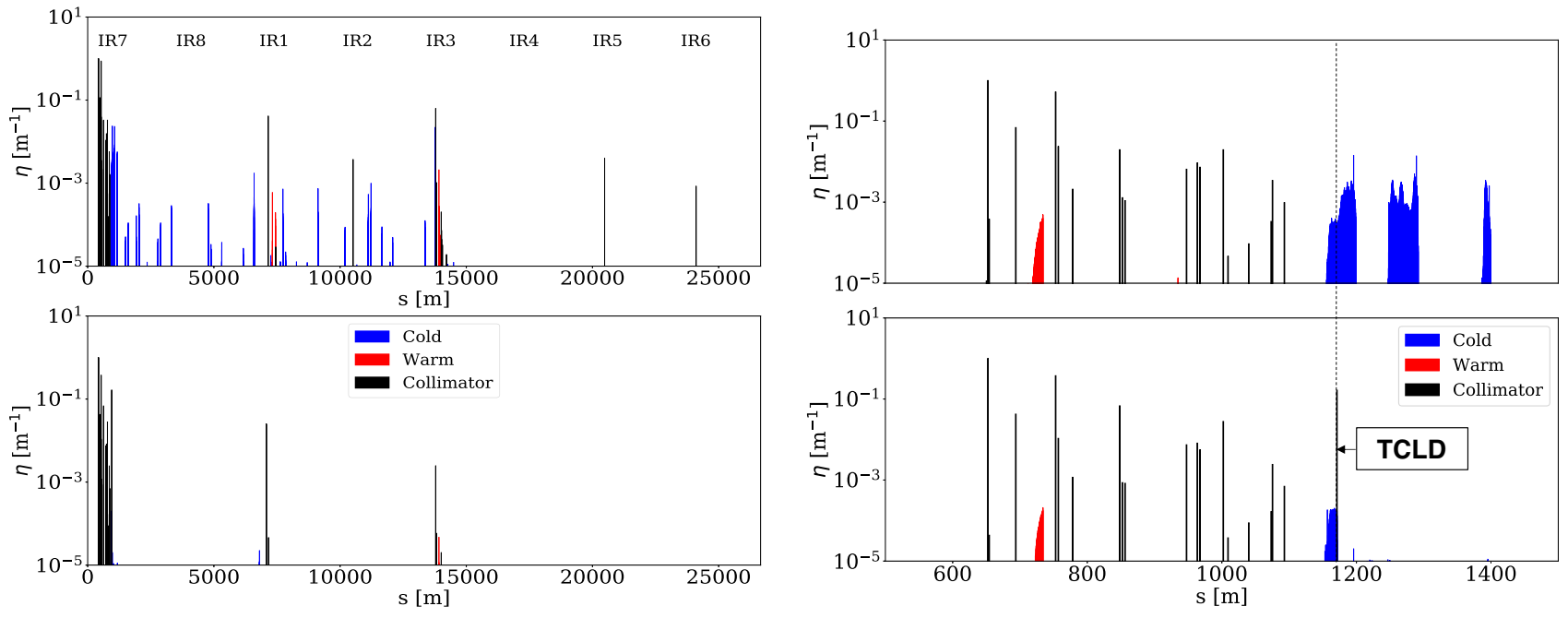

(a)

(b)

FIG. 17. Horizontal B1 full ring (a) and IR7 zoom (b) loss map for the 2018 LHC (top) and HL-LHC (bottom) ${ }^{208} \mathrm{~Pb}^{82+}$ ion operation layout. 

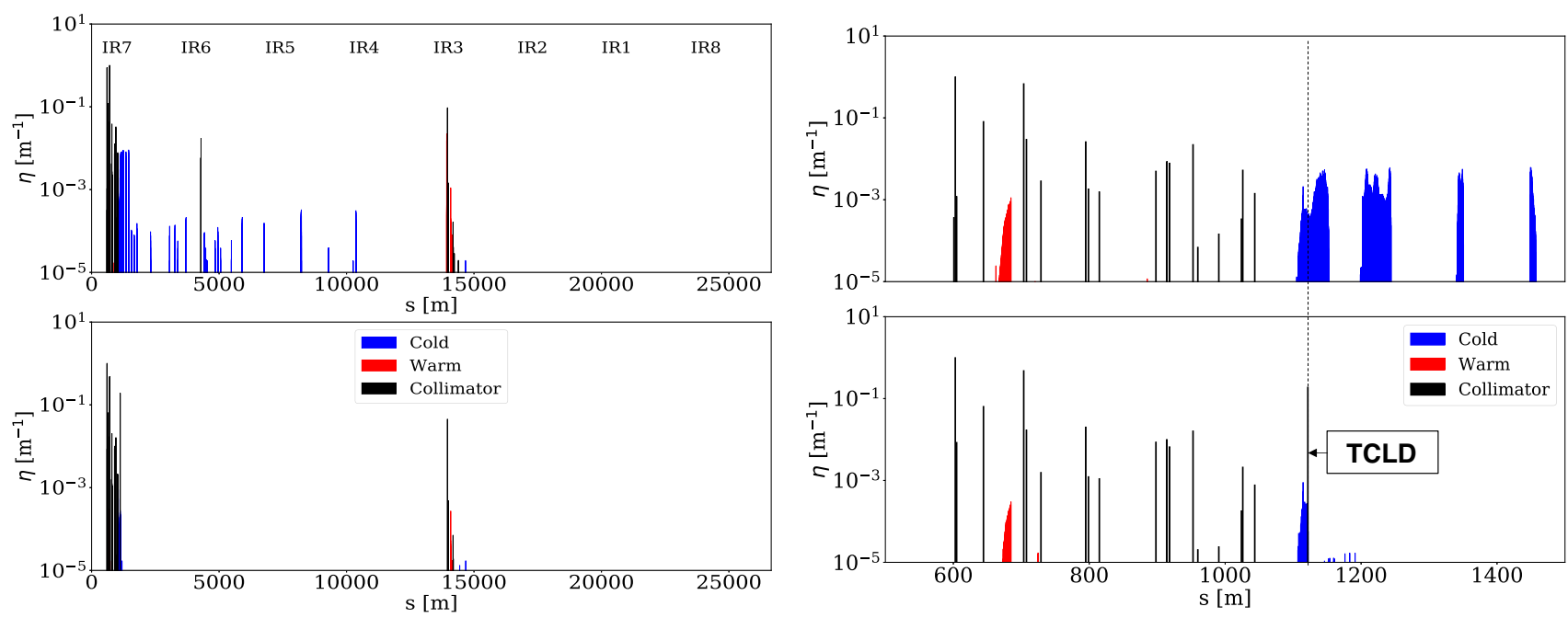

(a)

(b)

FIG. 18. Horizontal B2 full ring (a) and IR7 zoom (b) loss map for the LHC (top) and HL-LHC (bottom) ${ }^{208} \mathrm{~Pb}^{82+}$ ion operation layout.

\section{CONCLUSIONS}

A good understanding of heavy-ion collimation is essential to devise collimator settings providing safe operation, which is not interrupted by beam dumps or magnet quenches. In the short heavy-ion runs, the machine availability is crucial and every fill contributes significantly to the total integrated luminosity. Moreover, the collimation setup and validation should be done only once at the start of the run to avoid interruptions of the operation for physics. It is then very important to have accurate models to identify potential performance bottlenecks beforehand and to be able to react quickly in case of unexpected issues during the operation.

In the past ten years, a big effort has been made to improve the accuracy of the heavy-ion collimation simulation tools, combining reliable fragmentation models and precise tracking codes. This is a complex task because it requires putting together different tools optimized for different purposes. This effort has resulted in the development of the SIXTRACK-FLUKA coupling framework for heavy-ion beams, which has demonstrated its increasing reliability as a guide to understanding the origin and location of the losses in different scenarios such as standard collimation cleaning or dump failure scenarios.

For the $2018{ }^{208} \mathrm{~Pb}^{82+}$ ion run commissioning, the simulations were applied to mitigate losses through optimization of the collimator settings, and the results were confirmed by experimental studies. In general, very good qualitative agreement is observed in the losses at the collimators between our simulations and the measurements. The response of the BLMs could explain some of the observed discrepancies as well as machine imperfections, which are not taken into account in the simulations. In addition, the bottleneck location of losses in cold magnets is very well reproduced in the DS of IR7. Using these developed tools, effective mitigation strategies can be formulated and tested before implementation, given the predictive power of numerical simulations as shown for the 2018 ${ }^{208} \mathrm{~Pb}^{82+}$ ion run. The qualitative agreement between measurements and simulations is improved when detailed FLUKA energy deposition studies of secondary and tertiary particles are performed including the complete geometry of the accelerator and BLMs, and a quantitative agreement is found within a factor of a few pGy to over several hundreds of meters throughout the whole IR7. Given the complexity of the simulations, the fact that the losses span many orders of magnitude, and the many unknown error sources, we consider this a very good agreement. These results demonstrate the maturity of the simulation chain and give confidence in the simulations of future machine configurations.

The performance of the HL-LHC project collimation layout has been evaluated with the coupling simulation tool. The results show a very significant improvement with respect to the current LHC layout and no limitations for the heavy-ion run are predicted after the deployment of new DS collimators, whose location was optimized with the new tools described in this paper.

\section{ACKNOWLEDGMENTS}

This work was partially supported by the high-luminosity Large Hadron Collider project. The authors would like to thank colleagues in the collimation team for valuable discussions as well as the LHC operation team for their help in the acquisition of the loss maps analyzed in the paper. We express also our gratitude to J. Jowett and M. Schaumann for helpful discussions.

\section{APPENDIX A: ABBREVIATIONS}

In Table $\mathrm{V}$ the abbreviations used throughout the paper in alphabetic order are listed. 
TABLE V. Summary of abbreviations used in this paper.

\begin{tabular}{ll}
\hline \hline Abbreviations & Description \\
\hline ABDF & Asynchronous beam dump failure \\
B1, B2 & The two LHC counterrotating beams \\
BDF & Beam dump failure \\
BLM & Beam loss monitor \\
CFC & Carbon-fiber composite \\
DS & Dispersion suppressor \\
HL-LHC & High-luminosity Large Hadron Collider \\
IR & Insertion region. The LHC has eight IRs \\
LHC & Large Hadron Collider \\
MKD & Extraction kicker magnet in the beam dumping system \\
rf & Radio frequency \\
SC & Superconducting \\
SMPF & Single-module prefire \\
TCDQ & Large absorber used for beam dump protection in IR6 \\
TCL & Collimator for experiment debris in IR1, IR2, IR5 and IR8 \\
TCLA & Absorbers installed in IR7 and IR3 \\
TCLD & Dispersion suppressor collimator \\
TCP & Primary collimator installed in IR7 and IR3 \\
TCSG & Secondary collimator installed in IR7 and IR3 \\
TCSP & Secondary collimator used for beam dump protection in IR6 \\
TCTPH, TCTPV & Horizontal (H) and vertical (V) tertiary collimators located in the experiment IRs \\
\hline \hline
\end{tabular}

\section{APPENDIX B: TRACKING MAPS}

In the following text, we describe a generalized Hamiltonian for multi-isotopic particle beams used to derive the thin-lens symplectic tracking maps implemented in SIXTRACK.

Consider the trajectory of an arbitrary particle of rest mass $m$ and charge $Z e$ (with the charge multiplicity $Z$ and elementary charge $e$ ) moving at the relative speed $\beta=\frac{v}{c}$ through a dipolar magnetic field $B$. The trajectory is bent by a bending radius $\rho$, which is related to the magnetic field, the particle momentum, and charge as

$$
B \rho=\frac{P}{Z e} .
$$

The particle momentum can be written as $P=m \beta c \gamma$ with $\gamma$ the relativistic Lorentz factor normalized energy. The bending radius $\rho_{0}$ of the reference particle, with its physical properties defined by the parameters $m_{0}, Z_{0}, \beta_{0}$, is related to $\rho$ as follows:

$$
\frac{\rho}{\rho_{0}}=\frac{(1+\delta)}{\chi}, \quad \chi=\frac{m_{0}}{m} \frac{Z}{Z_{0}}, \quad(1+\delta)=\frac{\beta \gamma}{\beta_{0} \gamma_{0}} .
$$

The quantity $\chi$ defines the mass-to-charge ratio of the ion relative to the reference particle and the quantity $\delta$ is the relative offset of the normalized relativistic momentum.

Elementary transformations of Eq. (B2) show that $\delta$ in the multi-isotopic case is not the well-known relative momentum offset, but the relative momentum offset per mass unit

$$
\delta=\frac{P}{m} \frac{m_{0}}{P_{0}}-1
$$

For the case of heavy ions, the relative momentum offset of Eq. (B3) can be larger than 2 orders of magnitude. Both $\chi$ and $\delta$ quantify the dispersive offset of the particle trajectory acquired after interaction with the collimator material. Note that for the monoisotopic case when the same ions of the main beam are produced, $m \rightarrow m_{0}$ and $Z \rightarrow Z_{0}$, the two Eqs. (B2) and (B3) yield to the well-known expressions in which $\delta$ is the relative offset of the full momentum.

Consider a physical system described by the canonical coordinates $\mathbf{p}, \mathbf{q}$ with $\mathbf{p}=\left\{p_{x}, p_{y}, p_{z}\right\}$ and $\mathbf{q}=\{x, y, z\}$. After the transformation of the independent variable from $t$ to the path length $s(t)$, the accelerator Hamiltonian for the set of canonical variables $\left(x, p_{x}\right),\left(y, p_{y}\right),(-t, E)$ is given by [54]

$$
H=-p_{z}=-Z e A_{z}-\sqrt{\frac{(E-Z e \phi)^{2}}{c^{2}}-m^{2} c^{2}-\left(p_{x}-Z e A_{x}\right)^{2}-\left(p_{y}-Z e A_{y}\right)^{2}},
$$


where $\phi$ is the scalar potential, $A_{i}$ the electromagnetic vector potential, defining the magnetic field vector $\mathbf{B}=$ $\nabla \times \mathbf{A}$ and the canonical momenta $p_{i}$ are defined as

$$
p_{i}=m \gamma \dot{q}_{i}+Z e A_{i}
$$

The total particle energy $E$ in the square root of Eq. (B4) is a very large quantity. In order to solve analytically complicated vector potentials, the Hamiltonian should be expanded and this requires the dynamic variables in the square root to be small. The following substitutions of $E$, $p_{i}, A_{i}$ and $H$ serve the purpose of obtaining small dynamic variables in the square root of Eq. (B4), while maintaining the validity of Hamilton's equations:

$$
\begin{aligned}
p_{i} \rightarrow \tilde{p}_{i}=\frac{p_{i}}{P_{0}} \frac{m_{0}}{m} & H \rightarrow \tilde{H}=\frac{H}{P_{0}} \frac{m_{0}}{m}, \\
Z e A_{i} \rightarrow \chi a_{i}=\chi \frac{Z_{0} e A_{i}}{P_{0}} & E \rightarrow \tilde{E}=\frac{E}{P_{0}} \frac{m_{0}}{m} .
\end{aligned}
$$

The normalization with respect to the mass is essential to fulfill the requirement of obtaining small quantities in the square root of Eq. (B4) because the masses of the different ions produced by the interaction with collimators can differ significantly from the mass of the main ion beam. Note that the definition of the normalized vector potential $a_{i}$ is identical to the definition for the monoisotopic case [55]. Instead of incorporating it into the definition of $a_{i}$, the magnetic rigidity change for isotopes different from the reference particle is taken into account by the additional factor $\chi$. This allows the usage of well-known vector potentials from the derivation of the monoisotopic tracking maps [56,57].

Expressed in terms of the new coordinates, and assuming that a gauge can be found, such that $\phi=0$, and using the relativistic energy-momentum relation and Eq. (B3), the Hamiltonian can be written as

$$
\tilde{H}=-\chi a_{z}-\sqrt{(1+\delta)^{2}-\left(\tilde{p}_{x}-\chi a_{x}\right)^{2}-\left(\tilde{p}_{y}-\chi a_{y}\right)^{2}} .
$$

This equation is similar to the standard expression used in [57]. It should, however, be kept in mind that the quantities $p_{i}, \tilde{H}$ and $\delta$ are defined differently.
In order to also describe the longitudinal particle motion (e.g., the synchrotron motion) by small quantities, another transformation is required that can be obtained by means of a transformation of the canonical variables provided by a generating function of second type [55]

$$
F_{2}=x P_{x}+y P_{y}+\left(s-\beta_{0} c t\right)\left(p_{z}+\frac{E_{0}}{\beta_{0} P_{0} c}\right) .
$$

The old $\left(\tilde{p}_{i}, q_{i}\right)$ and new $\left(P_{i}, Q_{i}\right)$ coordinates, as well as the old $(\tilde{H})$ and new $(K)$ Hamiltonian are related by the following relations:

$\tilde{p}_{i}=\frac{\partial F_{2}}{\partial q_{i}} \quad Q_{i}=\frac{\partial F_{2}}{\partial P_{i}} \quad K=\tilde{H}+\frac{\partial F_{2}}{\partial s}=\tilde{H}+p_{z}$.

The transformed variables $\left(X, P_{x}\right),\left(Y, P_{y}\right),\left(z, P_{z}\right)$ are then defined as follows:

$$
\begin{array}{r}
X=x, \quad Y=y, \quad z=s-\beta_{0} c t, \\
P_{x}=\tilde{p}_{x}, \quad P_{y}=\tilde{p}_{y}, \quad p_{z}=\frac{\frac{m_{0}}{m} E-E_{0}}{\beta_{0} P_{0} c} .
\end{array}
$$

Including a last transformation for convenience $P_{i} \rightarrow p_{i}$, $K \rightarrow H$, the final multi-isotopic Hamiltonian in a straight coordinate system yields

$H=p_{z}-\sqrt{(1+\delta)^{2}-\left(p_{x}-\chi a_{x}\right)^{2}-\left(p_{y}-\chi a_{y}\right)^{2}}-\chi a_{z}$.

The new coordinate $z$ describes the difference in arrival time with respect to the reference particle. The quantity $p_{z}$ is the canonical conjugate of $z$.

To describe particle motion in an accelerator it is convenient to transform the straight coordinate system into a curved one, with the new set of variables $\left(X, P_{x}\right)$, $\left(Y, P_{y}\right),\left(s, P_{s}\right)$. In a coordinate system horizontally bent by a constant radius $\rho_{0}=1 / h_{x}$, the Hamiltonian becomes

$$
H=p_{z}-\left[1-h_{x}(s) x\right]\left\{\sqrt{(1+\delta)^{2}-\left[p_{x}-\chi a_{x}(s)\right]^{2}-\left[p_{y}-\chi a_{y}(s)\right]^{2}}+\chi a_{s}(s)\right\}
$$

where $a_{s}$ is the vector potential in the curvilinear reference coordinates. The full derivation can be found in [14]. In the monoisotopic limit $m \rightarrow m_{0}$ and $Z \rightarrow Z_{0}$ the multi-isotopic Hamiltonian becomes the standard Hamiltonian presented in $[55,58]$. 
Depending on the complexity of the electromagnetic field of the beam line element and the corresponding boundary conditions it is useful to expand the square root of the Hamiltonian in Eq. (B14) in $\frac{\left(p_{x}-\chi a_{x}\right)^{2}+\left(p_{y}-\chi a_{y}\right)^{2}}{(1+\delta)^{2}}$ to first order, as it is done for monoisotopic beams in [54], and the Hamiltonian becomes

$$
H \approx p_{z}-\left[1-h_{x}(s) x\right]\left[(1+\delta)\left(1-\frac{1}{2} \frac{\left[p_{x}-\chi a_{x}(s)\right]^{2}+\left[p_{y}-\chi a_{y}(s)\right]^{2}}{(1+\delta)^{2}}\right)+\chi a_{s}(s)\right]
$$

The accuracy of the tracking maps derived using the expanded Hamiltonian in Eq. (B15) was studied for the drift space in the monoisotopic case. The results using the exact Hamiltonian are in very good agreement if $p_{x}$ and $p_{y}$ are small and significant differences arise only if these values are so large that the particles would be lost in the magnet aperture after only a few meters [14]. For the driftspace element, both tracking maps derived from the exact and the expanded multi-isotopic accelerator Hamiltonian in the thin-lens approximation were implemented in SIXTRACK. The user can chose which option should be used depending on the requirements on the simulations precision and time. No significant increase in CPU time is expected if the number of simulated turns is smaller than $10^{5}$ [59]. Based on simulation studies a value of 700 turns have been defined and used in all cases presented in this paper, for which all simulated particles are lost in the ring. For the other beam line elements only the expanded Hamiltonian tracking maps were implemented because under the thinlens approximation higher order terms cancel and the resulting tracking maps from one or the other Hamiltonian are equivalent. The symplecticity of the tracking maps was demonstrated by the means of the Jacobian matrix and the details of the derivation can be found in [14].

In the following, the developed tracking maps from the approximated multi-isotopic Hamiltonian for the different accelerator elements are described. Notice that in SiXTRACK instead of the transverse canonical momenta $p_{x}$ and $p_{y}$ the evolution of $x^{\prime}$ and $y^{\prime}$ is computed. As examples, the tracking maps implemented in SIXTRACK for a thin-lens kicker dipole and the quadrupole are also presented. Further details on the implementation in SIXTRACK are given in [14].

\section{Drift space}

A drift space is defined by the absence of electromagnetic fields $a_{i}=0$. The ideal trajectory is not bent, thus $h_{x}=0$ and the expanded Hamiltonian yields

$$
H \approx p_{\sigma}-\delta+\frac{1}{2} \frac{p_{x}^{2}+p_{y}^{2}}{(1+\delta)}
$$

The resulting tracking maps are independent of the ion species and thus identical to the monoisotopic case. The resulting tracking maps are

$$
\begin{gathered}
\left(\begin{array}{c}
x^{F} \\
y^{F} \\
z^{F}
\end{array}\right)=\left(\begin{array}{c}
x^{I}+\frac{p_{x}^{I}}{1+\delta} L \\
y^{I}+\frac{p_{y}^{I}}{1+\delta} L \\
z^{I}-L \frac{\beta_{0}}{\beta_{z}}\left(1+\frac{1}{2} \frac{\left(p_{x}^{I}\right)^{2}+\left(p_{y}^{I}\right)^{2}}{(1+\delta)^{2}}\right)
\end{array}\right) \\
\left(\begin{array}{c}
p_{x}^{F} \\
p_{y}^{F} \\
p_{z}^{F}
\end{array}\right)=\left(\begin{array}{c}
p_{x}^{I} \\
p_{y}^{I} \\
p_{z}^{I}
\end{array}\right)
\end{gathered}
$$

\section{Dipole magnet}

\section{a. Bending dipole}

Using the vector potential of a bending dipole derived in [56]

$$
a_{x}=a_{y}=0, \quad a_{s}=k_{0}\left(x+\frac{h_{x} x^{2}}{2}\right),
$$

the expanded Hamiltonian for a horizontal bending dipole magnet with the normalized strength $k_{0}=\frac{B_{y} Z_{0} e}{P_{0}}$ and $h_{x} \neq 0$ is given by

$$
\begin{aligned}
H \approx & p_{\sigma}-\left(1+h_{x} x\right)(1+\delta) \\
& +\frac{1}{2} \frac{p_{x}^{2}+p_{y}^{2}}{(1+\delta)}+\chi k_{0}\left(x+\frac{h_{x} x^{2}}{2}\right) .
\end{aligned}
$$

The tracking map for a dipole of length $L$ in thin-lens approximation $k_{0} L \rightarrow 0$ derived using Hamilton's equations is

$$
\begin{gathered}
\left(\begin{array}{c}
x^{F} \\
y^{F} \\
z^{F}
\end{array}\right)=\left(\begin{array}{c}
x^{I} \\
y^{I} \\
z^{I}
\end{array}\right) \\
\left(\begin{array}{c}
p_{x}^{F} \\
p_{y}^{F} \\
p_{z}^{F}
\end{array}\right)=\left(\begin{array}{c}
p_{x}^{I}+L\left[h_{x}(1+\delta)-k_{0} \chi\left(1+h_{x} x^{I}\right)\right] \\
p_{y}^{I} \\
p_{z}^{I}
\end{array}\right) .
\end{gathered}
$$




\section{b. Kicker dipole}

The magnetic kicker dipole provides a transverse magnetic field, similar to the bending dipole, but the reference orbit is not bent $\left(h_{x}=0\right)$. Kicker dipoles are used to control the orbit in a machine. From the Hamiltonian in Eq. (B20) with $h_{x}=0$, the resulting tracking maps are

$$
\begin{gathered}
\left(\begin{array}{c}
x^{F} \\
y^{F} \\
z^{F}
\end{array}\right)=\left(\begin{array}{c}
x^{I} \\
y^{I} \\
z^{I}
\end{array}\right) \\
\left(\begin{array}{c}
p_{x}^{F} \\
p_{y}^{F} \\
p_{z}^{F}
\end{array}\right)=\left(\begin{array}{c}
p_{x}-k_{0} \chi L \\
p_{y}^{I} \\
p_{z}^{I}
\end{array}\right) .
\end{gathered}
$$

Taking into account that $x^{\prime}=\frac{p_{x}}{(1+\delta)}$, the transformation of $x^{\prime}$ yields

$$
\left(x^{\prime}\right)^{F}=\left(x^{\prime}\right)^{I}-k_{0} L \frac{\chi}{(1+\delta)} .
$$

\section{Quadrupole}

The quadrupole magnets are used to provide focusing in order to confine the transverse dimension of the beam. The vector potential of a horizontal or vertical quadrupole magnet in normalized coordinates is given by

$$
a_{x}=0, \quad a_{y}=0, \quad a_{s}=-\frac{1}{2} k\left(y^{2}-x^{2}\right),
$$

where $k=\frac{q_{0}}{P_{0}} g$ is the normalized quadrupole gradient which has the unit of $m^{2}$ and $g$ is the quadrupole gradient. The following Hamiltonian can be derived to describe the quadrupole in thin-lens approximation,

$$
H \approx H_{D}+\frac{1}{2} \tilde{\delta}\left(s-s_{0}\right) L \chi k\left(x^{2}-y^{2}\right),
$$

where $H_{D}$ is the approximated Hamiltonian for a drift space. The tracking maps for a thin-lens quadrupole are given by

$$
\begin{gathered}
\left(\begin{array}{c}
x^{F} \\
y^{F} \\
z^{F}
\end{array}\right)=\left(\begin{array}{c}
x^{I} \\
y^{I} \\
z^{I}
\end{array}\right) \\
\left(\begin{array}{c}
p_{x}^{F} \\
p_{y}^{F} \\
p_{z}^{F}
\end{array}\right)=\left(\begin{array}{c}
p_{x}^{I}-\chi k L x^{I} \\
p_{y}^{I}+\chi k L y^{I} \\
p_{z}^{I}
\end{array}\right) .
\end{gathered}
$$

This transfer map corresponds to a focusing lens in the horizontal and defocusing lens in the vertical direction. The transformation of $x^{\prime}$ and $y^{\prime}$ is given by

$$
\left(\begin{array}{c}
\left(x^{\prime}\right)^{F} \\
\left(y^{\prime}\right)^{F}
\end{array}\right)=\left(\begin{array}{c}
\left(x^{\prime}\right)^{I}-k L x^{I} \frac{\chi}{1+\delta} \\
\left(y^{\prime}\right)^{I}+k L y^{I} \frac{\chi}{1+\delta}
\end{array}\right) .
$$

\section{Accelerating rf cavity}

The energy gain $\Delta E$ of a particle in an accelerating cavity with wave number $k=\frac{\omega}{c}=2 \pi f$ can be approximated by

$$
\Delta E=Z e U \sin \left(\phi-k \frac{\sigma}{\beta_{0}}\right)
$$

where $U$ is the average voltage during the particle's passage through the cavity [55]. In the approximation of a thin cavity, the following vector potential can be derived:

$$
A_{x}=A_{y}=0 \quad A_{s}=-\frac{U}{\omega} \cos \left(\phi-k \frac{\sigma}{\beta_{0}}\right) \tilde{\delta}(s),
$$

where $\tilde{\delta}(s)$ is the Dirac function. Using the substitution $U_{n}=\frac{Z_{0} e}{P_{0} c} U$, the transfer map for $p_{z}$ can be deduced.

The resulting expanded Hamiltonian for a thin cavity is then given by

$H \approx H_{D}+\chi q_{0} \frac{U C}{\beta_{0}^{2} E_{0} 2 \pi h} \cos \left(\frac{2 \pi h}{C} \sigma+\phi\right) L \tilde{\delta}\left(s-s_{0}\right)$.

The thin-lens tracking maps for the accelerating rf cavity are the following:

$$
\begin{gathered}
\left(\begin{array}{c}
x^{F} \\
y^{F} \\
z^{F}
\end{array}\right)=\left(\begin{array}{c}
x^{I} \\
y^{I} \\
z^{I}
\end{array}\right) \\
\left(\begin{array}{c}
p_{x}^{F} \\
p_{y}^{F} \\
p_{z}^{F}
\end{array}\right)=\left(\begin{array}{c}
p_{x}^{I} \\
p_{y}^{I} \\
p_{z}^{I}+\chi q_{0} \frac{1}{\beta_{0}^{2}} \frac{U}{E_{0}} L \sin \left(\frac{2 \pi h}{C}+\phi\right)
\end{array}\right) .
\end{gathered}
$$

The change in $p_{\sigma}$ is, as expected, proportional to $q \frac{m_{0}}{m}$ by the relation between $p_{\sigma}$ and $E$, the energy transfer by the $\mathrm{rf}$ cavity of length $L$ corresponds to the expression given in Eq. (B31) and yields

$$
\delta E=q U L \sin \left(\frac{2 \pi h}{C}+\phi\right) .
$$

\section{Thin multipole}

Higher order magnetic fields are described in a more generic way as 


$$
B_{y}+i B_{x}=\sum_{n=1}^{\infty}\left(b_{n}+i a_{n}\right)\left(\frac{(x+i y)^{n}}{r_{0}^{n-1}}\right) .
$$

In this context, $n$ is the multiple order, $b_{n}, a_{n}$ are the multiple coefficients, one component is for the upright fields and the other one is for the slanted ones, which describe the field orientation for the contribution of each multiple order. The quantity $r_{0}$ is a reference radius. The magnetic field described in Eq. (B37) corresponds to the following vector potential:

$A_{x}=0, \quad A_{y}=0, \quad A_{z}=-\operatorname{Re} \sum_{n=1}^{\infty}\left(b_{n}+i a_{n}\right)\left(\frac{x+i y}{r_{0}}\right)^{n-1}$.

Inserting this vector potential into the Hamiltonian in thinlens approximation yields

$H \approx H_{D}-\frac{q_{0}}{P_{0}} \chi L \tilde{\delta}\left(s-s_{0}\right) \operatorname{Re} \sum_{n=1}^{\infty}\left(b_{n}+i a_{n}\right)\left(\frac{x+i y}{r_{0}}\right)^{n-1}$.

The tracking maps for the thin multiple kick

$$
\begin{aligned}
& \left(\begin{array}{c}
x^{F} \\
y^{F} \\
z^{F}
\end{array}\right)=\left(\begin{array}{c}
x^{I} \\
y^{I} \\
z^{I}
\end{array}\right) \\
& \left(\begin{array}{c}
p_{x}^{F} \\
p_{y}^{F} \\
p_{z}^{F}
\end{array}\right)= \\
& \left(\begin{array}{c}
\left.p_{x}^{I}-\chi L \operatorname{Re}\left[\sum_{n=1}^{\infty}\left(k_{n}+i \hat{k_{n}}\right)(x+i y)^{n-1}\right)\right] \\
\left.p_{y}^{I}-\chi L \operatorname{Re}\left[\sum_{n=1}^{\infty}\left(k_{n}+i \hat{k_{n}}\right)(x+i y)^{n-1}\right)\right] \\
p_{z}^{I}
\end{array}\right),
\end{aligned}
$$

where $k_{n}$ and $\hat{k_{n}}$ are defined as

$$
k_{n}=\frac{q_{0}}{P_{0}} \frac{a_{n}}{r_{0}^{n-1}} \quad \text { and } \quad \hat{k_{n}}=\frac{q_{0}}{P_{0}} \frac{b_{n}}{r_{0}^{n-1}} .
$$

[1] O. S. Brüning, P. Collier, P. Lebrun, S. Myers, R. Ostojic, J. Poole, and P. Proudlock, LHC Design Report, CERN Yellow Reports: Monographs (CERN, Geneva, 2004).

[2] R.W. Assmann, Collimators and beam absorbers for cleaning and machine protection, in Proceedings of the
LHC Project Workshop Chamonix XIV (CERN, Geneva, 2005).

[3] G. Robert-Demolaize, Design and performance optimization of the LHC collimation system, Ph.D thesis, presented in Grenoble, France, 2006.

[4] R.W. Assmann et al., The final collimation system for the LHC, in Proceedings of the 10th European Particle Accelerator Conference, Edinburgh, Scotland (2006).

[5] C. Bracco, Commissioning scenarios and tests for the LHC collimation system, Ph.D thesis, presented in Lausanne, Switzerland, 2008.

[6] R. Bruce et al., Simulations and measurements of beam loss patterns at the CERN Large Hadron Collider, Phys. Rev. Accel. Beams 17, 081004 (2014).

[7] R. Bruce, R. Assmann, V. Boccone, G. Bregliozzi, H. Burkhardt, F. Cerutti, A. Ferrari, M. Huhtinen, A. Lechner, Y. Levinsen, A. Mereghetti, N. Mokhov, I. Tropin, and V. Vlachoudis, Sources of machine-induced background in the ATLAS and CMS detectors at the CERN Large Hadron Collider, Nucl. Instrum. Methods Phys. Res., Sect. A 729, 825 (2013).

[8] R. Bruce, M. Huhtinen, A. Manousos, F. Cerutti, L. Esposito, R. Kwee-Hinzmann, A. Lechner, A. Mereghetti, D. Mirarchi, S. Redaelli, and B. Salvachua, Collimationinduced experimental background studies at the CERN Large Hadron Collider, Phys. Rev. Accel. Beams 22, 021004 (2019).

[9] H. H. Braun et al., Collimation of heavy ion beams in the LHC, in Proceedings of the 9th European Particle Accelerator Conference, Lucerne, 2004 (EPS-AG, Lucerne, 2004), p. 551.

[10] P. Hermes, R. Bruce, J. Jowett, S. Redaelli, B. S. Ferrando, G. Valentino, and D. Wollmann, Measured and simulated heavy-ion beam loss patterns at the CERN Large Hadron Collider, Nucl. Instrum. Methods Phys. Res., Sect. A 819, 73 (2016).

[11] H.H. Braun et al., Hadronic and electromagnetic fragmentation of ultrarelativistic heavy ions at LHC, Elsevier report, 2004.

[12] P. Hermes et al., Simulation tools for heavy-ion tracking collimation, available at https://cds.cern.ch/record/ 2646800.

[13] J. M. Jowett, H. H. Braun, M. I. Gresham, E. Mahner, A. N. Nicholson, and E. Shaposhnikova, Limits to the performance of the LHC with ion beams, in Proceedings of the 9th European Particle Accelerator Conference, Lucerne, 2004 (Ref. [9]), p. 578.

[14] P. D. Hermes, Heavy-ion collimation at the Large Hadron Collider: Simulations and measurements, Ph.D. thesis, University of Munster, 2016, available at https://cds.cern .ch/record/2241364.

[15] N. Fuster-Martínez et al., Run 2 collimation overview, in Proceedings of the 9th Evian Workshop, 2019 (to be published).

[16] J. Jowett et al., Overview of heavy-ions in LHC Run 2, 9th LHC Operations Evian Workshop (to be published).

[17] J. Jowett et al., The 2018 heavy-ion run of the LHC, in Proceedings of the 10th International Particle Accelerator Conference (IPAC2019), Melbourne, Australia (2019), WEYYPLM2. 
[18] J. Jowett, R. Alemany-Fernandez, R. Bruce, M. Giovannozzi, P. Hermes, W. Höfle, M. Lamont, T. Mertens, S. Redaelli, M. Schaumann, J. Uythoven, and J. Wenninger, The 2015 heavy-ion run of the LHC, in Proceedings of the 7th International Particle Accelerator Conference (IPAC2016), Busan, South Korea (2016), TUPMW027.

[19] D. Mirarchi et al., Special losses, in Proceedings of the 9th Evian Workshop, 2019 (to be published).

[20] G. Apollinari, I. B. Alonso, and O. Brüning, P. Fessia, M. Lamont, L. Rossi, and L. Tavian, High-Luminosity Large Hadron Collider (HL-LHC): Technical Design Report V. 0.1, CERN Yellow Reports: Monographs (CERN, Geneva, 2017), available at https://cds.cern.ch/record/2284929.

[21] R. De Maria et al., SIXTRACK version 5: Status and new developments, in Proceedings of the 10th International Particle Accelerator Conference (IPAC'19) (2019), WEPTS043, available at http://jacow.org/ipac2019/papers/ wepts043.pdf.

[22] SIXTRACK website, http://sixtrack.web.cern.ch/SixTrack/.

[23] A. Ferrari, P. R. Sala, A. Fasso, and J. Ranft, FLuKA: A multiparticle transport code (program version 2005), available at http://cds.cern.ch/record/898301/files/CERN-2005010.pdf.

[24] T. Bohlen, F. Cerutti, M. Chin, A. Fassò, A. Ferrari, P. Ortega, A. Mairani, P. Sala, G. Smirnov, and V. Vlachoudis, The FLUKA code: Developments and challenges for high energy and medical applications, Nucl. Data Sheets 120, 211 (2014).

[25] A. Mereghetti et al., SixTrack-FLUKA active coupling for the upgrade of the SPS scrapers, in Proceedings of the 4th International Particle Accelerator Conference, IPAC2013, Shanghai, China (JACoW, Shanghai, China, 2013), p. 2657.

[26] E. Skordis, V. Vlachoudis, R. Bruce, F. Cerutti, A. Ferrari, A. Lechner, A. Mereghetti, P. Ortega, S. Redaelli, and D. S. Pastor, FLUKA coupling to SIXTRACK, in Proceedings of the ICFA Mini-Workshop on Tracking for Collimation (CERN, Geneva, Switzerland, 2018), p. 17.

[27] R. Assmann, B. Goddard, E. Vossenberg, and E. Weisse, The consequences of abnormal beam dump actions on the LHC collimation system, LHC Project Note 293, CERN, 1996.

[28] R. Schmidt, R. Assmann, E. Carlier, B. Dehning, R. Denz, B. Goddard, E. B. Holzer, V. Kain, B. Puccio, B. Todd, J. Uythoven, J. Wenninger, and M. Zerlauth, Protection of the CERN Large Hadron Collider, New J. Phys. 8, 290 (2006).

[29] R. Bruce, R. W. Assmann, and S. Redaelli, Calculations of safe collimator settings and $\beta^{*}$ at the CERN Large Hadron Collider, Phys. Rev. Accel. Beams 18, 061001 (2015).

[30] R. Bruce, C. Bracco, R. D. Maria, M. Giovannozzi, A. Mereghetti, D. Mirarchi, S. Redaelli, E. Quaranta, and B. Salvachua, Reaching record-low $\beta^{*}$ at the CERN Large Hadron Collider using a novel scheme of collimator settings and optics, Nucl. Instrum. Methods Phys. Res., Sect. A 848, 19 (2017).

[31] G. Azzopardi, B. Salvachua, and G. Valentino, Data-driven cross-talk modeling of beam losses in LHC collimators, Phys. Rev. Accel. Beams 22, 083002 (2019).

[32] W. Hofle, R. Assmann, S. Redaelli, R. Schmidt, D. Valuch, D. Wollmann, and M. Zerlauth, Controlled transverse blowup of high-energy proton beams for aperture measurements and loss maps, in Proceedings of the $3 \mathrm{rd}$ International Particle Accelerator Conference, New1441Orleans, LA, 2012 (IEEE, Piscataway, NJ, 2012), p. 4059.

[33] E. Holzer, B. Dehning, E. Effinger, J. Emery, G. Ferioli, J. Gonzalez, E. Gschwendtner, G. Guaglio, M. Hodgson, D. Kramer, R. Leitner, L. Ponce, V. Prieto, M. Stockner, and C. Zamantzas, Beam loss monitoring system for the LHC, IEEE Nucl. Sci. Symp. Conf. Record 2, 1052 (2005), https://cds.cern.ch/record/930275/files/ab-2006-009.pdf.

[34] E. B. Holzer et al., Development, production and testing of 4500 beam loss monitors, in Proceedings of the 11th European Particle Accelerator Conference, Genoa, 2008 (EPS-AG, Genoa, Italy, 2008), p. 1134.

[35] P. Hermes, B. Auchmann, R. Bruce, W. Hofle, E. B. Holzer, M. Kalliokoski, G. Kotzian, A. Mereghetti, D. Mirarchi, E. Quaranta, S. Redaelli, B. S. Ferrando, G. Valentino, D. Valuch, D. Wollmann, and M. Zerlauth, LHC heavy-ion collimation quench test at $6.37 \mathrm{Z} \mathrm{TeV}$, Report No. CERN-ACC-NOTE-2016-0031, 2016.

[36] N. Holden, Development of the ICOSIM program and application to magnetized collimators in the LHC, Report No. CERN-AB-NOTE-2008-054, 2008.

[37] FLUKA website, http://www.fluka.org/fluka.php.

[38] H. H. Braun, A. Fassò, A. Ferrari, J. M. Jowett, P. R. Sala, and G. I. Smirnov, Hadronic and electromagnetic fragmentation of ultrarelativistic heavy ions at LHC, Phys. Rev. Accel. Beams 17, 021006 (2014).

[39] K. Sjobak, H. Burkhardt, R. D. Maria, A. Mereghetti, and A.S. Garca, General functionality for turn-dependent element properties in SIXTRACK, in Proceedings of the 6th International Particle Accelerator Conference, May 2015, Richmond, EEUU (2015), p. 468.

[40] K. Sjobak et al., Dynamic simulations in SixTrack, in Proceedings of the Tracking for Collimation Workshop (CERN, Geneva, Switzerland, 2015).

[41] M. Fraser, W. Bartmann, C. Bracco, E. Carlier, B. Goddard, V. Kain, N. Magnin, J. Uythoven, and F. Velotti, A beam-based measurement of the LHC beam dump kicker waveform, in Proceedings of the 7th International Particle Accelerator Conference, May 2016, Busan, South Korea (2016), p. 3911.

[42] R. W. Assmann, B. Goddard, E. B. Vossenberg, and E. Weisse, The consequences of abnormal beam dump actions on the LHC collimation system, Technical Report No. LHC-PROJECT-NOTE-293, CERN, Geneva, 2002.

[43] N. Fuster-Martinez, J. M. Jowett, R. Bruce, D. Mirarchi, and S. Redaelli, Performance of the collimation system during the 2018 lead ion run at the large hadron collider, in Proceedings of the 10th International Particle Accelerator Conference, May 2019, Melbourne, Australia (2019).

[44] E. Quaranta, A. Bertarelli, R. Bruce, F. Carra, F. Cerutti, A. Lechner, S. Redaelli, E. Skordis, and P. Gradassi, Modeling of beam-induced damage of the LHC tertiary collimators, Phys. Rev. Accel. Beams 20, 091002 (2017).

[45] A. Lechner, B. Auchmann, T. Baer, C. Bahamonde Castro, R. Bruce, F. Cerutti, L. S. Esposito, A. Ferrari, J. M. Jowett, A. Mereghetti, F. Pietropaolo, S. Redaelli, B. Salvachua, M. Sapinski, M. Schaumann, N. V. Shetty, V. Vlachoudis, and E. Skordis, Validation of energy deposition simulations 
for proton and heavy ion losses in the CERN Large Hadron Collider, Phys. Rev. Accel. Beams 22, 071003 (2019).

[46] E. Skordis, Radiation impact of collimation beam losses in the LHC and HL-LHC, Ph.D. Thesis, University of Liverpool, 2020.

[47] E. Skordis et al., Study of the 2015 top energy LHC collimation quench tests through an advanced simulation chain, in Proceedings of the 8th International Particle Accelerator Conference, May 2017, Copenhagen, Denmark (2017), MOPAB012, pp. 100-103.

[48] B. Salvachua-Ferrando et al., Cleaning performance of the LHC collimation system up to $4 \mathrm{TeV}$, in Proceedings of the 4th International Particle Accelerator Conference, May 2013, Shanghai, China (2013), MOPWO048.

[49] High-Luminosity Large Hadron Collider (HL-LHC): Technical design report V. 0.1, edited by G. Apollinari, I. B. Alonso, O. Bruning, P. Fessia, M. Lamont, L. Rossi, and L. Tavian, CERN Report No. CERN-2017-007-M, CERN, Geneva, 2017.

[50] L. Rossi and O. Brüning, Progress with the high luminosity LHC program at CERN, in Proceedings of the 10th International Particle Accelerator Conference, May 2019, Melbourne, Australia (2019), MOYPLM3.

[51] LHC injectors upgrade, edited by J. Coupard, H. Damerau, A. Funken, R. Garoby, S. Gilardoni, B. Goddard, K. Hanke, A. Lombardi, D. Manglunki, M. Meddahi,
B. Mikulec, G. Rumolo, E. Shaposhnikova, and M. Vretenar, Technical Design Report No. CERN-ACC2014-0337, 2014.

[52] C. Bahamonte et al., Energy deposition from collimation losses in the DS region at P7, at the 8th HL-LHC collaboration meeting, CERN (2018).

[53] F. Savary et al., Status of the $11 \mathrm{~T} \mathrm{Nb}_{3} \mathrm{Sn}$ dipole project for the LHC, IEEE Trans. Appl. Supercond. 25, 1 (2015), https://indico.cern.ch/event/742082/contributions/3085132/ attachments/1735864/2808038/Energy_deposition_from_ collimation_losses_in_the_DS_region_at_P7.pdf.

[54] E. Courant and H. Snyder, Theory of the alternatinggradient synchrotron, Ann. Phys. (N.Y.) 281, 360 (2000).

[55] A. Wolski, Beam Dynamics in High Energy Particle Accelerators (World Scientific, Singapore, 2014).

[56] D. P. Barber et al., A nonlinear canonical formalism for the coupled synchro-betatron motion of protons with arbitrary energy, DESY Technical Report No. 87-36, 1987.

[57] K. Heinemann et al., Construction of nonlinear symplectic six-dimensional thin-lens maps by exponentiation, DESY Technical Report No. 95-189, 1995.

[58] S. Turner, Physics course, Report No. CERN-95-06, 1995.

[59] M. Fjellstrom, Particle Tracking in Circular Accelerators Using the Exact Hamiltonian in SIXTRACK, Master thesis, Lulea University, Sweden, 2013. 